\title{
Performance Analysis of ZF and MMSE Equalizers for MIMO Systems: An In-Depth Study of the High SNR Regime
}

\author{
Yi Jiang Mahesh K. Varanasi Jian Li
}

\begin{abstract}
This paper presents an in-depth analysis of the zero forcing (ZF) and minimum mean squared error (MMSE) equalizers applied to wireless multi-input multi-output (MIMO) systems with no fewer receive than transmit antennas. In spite of much prior work on this subject, we reveal several new and surprising analytical results in terms of the well-known performance metrics of output signal-to-noise ratio (SNR), uncoded error and outage probabilities, diversity-multiplexing (D-M) gain tradeoff, and coding gain. Contrary to the common perception that ZF and MMSE are asymptotically equivalent at high SNR, we show that the output SNR of the MMSE equalizer (conditioned on the channel realization) is $\rho_{\mathrm{mmse}}=\rho_{\mathrm{zf}}+\eta_{\mathrm{snr}}$, where $\rho_{\mathrm{zf}}$ is the output SNR of the ZF equalizer, and that the gap $\eta_{\text {snr }}$ is statistically independent of $\rho_{\text {zf }}$ and is a non-decreasing function of input SNR. Furthermore, as snr $\rightarrow \infty, \eta_{\text {snr }}$ converges with probability one to a scaled $\mathcal{F}$ random variable. It is also shown that at the output of the MMSE equalizer, the interference-to-noise ratio (INR) is tightly upper bounded by $\frac{\eta_{\mathrm{snr}}}{\rho_{\mathrm{zf}}}$. Using the decomposition of the output SNR of MMSE, we can approximate its uncoded error as well as outage probabilities through a numerical integral which accurately reflects the respective SNR gains of the MMSE equalizer relative to its ZF counterpart. The $\epsilon$-outage capacities of the two equalizers, however, coincide in the asymptotically high SNR regime, despite the non-vanishing gap $\eta_{\text {snr }}$. By analyzing a fictitious parallel channel model with coding across the sub-channels in terms of the diversity-multiplexing (D-M) gain tradeoff, we provide the solution to a long-standing open problem: applying optimal detection ordering does not improve the D-M tradeoff of the V-BLAST (vertical Bell Labs layered Space-Time) architecture. However, by deriving tight lower bounds to the outage probabilities of ZF and MMSE equalizers, we show that optimal ordering yields a SNR gain of $10 \log _{10} N \mathrm{~dB}$ in the ZF-V-BLAST architecture (where $N$ is the number of transmit antennas) whereas for the MMSE-V-BLAST architecture, the SNR gain due to ordered detection is even better, and significantly so.
\end{abstract}

\section{Keywords}

This work was supported in part by the National Science Foundation Grant CCF-0423842 and CCF-0434410. This paper was presented in part at Globecom 2005.

Y. Jiang and M. Varanasi are with the Department of Electrical and Computer Engineering, University of Colorado, Boulder CO 80309, USA. Email: yjiang.ee@gmail.com, varanasi@colorado.edu

J. Li is with the Department of Electrical and Computer Engineering, University of Florida, Gainesville, FL 32611-6130, USA. Email: li@dsp.ufl.edu 
Zero forcing, minimum mean squared error, MIMO, error probability, V-BLAST, diversity gain, spatial multiplexing gain, tradeoff, outage capacity, outage probability. 


\section{INTRODUCTION}

Consider the complex baseband model for the wireless multi-input multi-output (MIMO) channel with $N$ transmit antennas and $M$ receiver antennas

$$
\mathbf{y}=\mathbf{H x}+\mathbf{z},
$$

where $\mathbf{y} \in \mathbb{C}^{M \times 1}$ is the received signal and $\mathbf{H} \in \mathbb{C}^{M \times N}$ is a Rayleigh fading channel with independent, identically distributed (i.i.d.), circularly symmetric standard complex Gaussian entries, denoted as $h_{i j} \sim N(0,1)$ for $1 \leq i \leq M, 1 \leq j \leq N$. We assume that the number of receive antennas is no less than the number of transmit antennas $(M \geq N)$. We also assume that the $N$ data substreams have uniform power, i.e., $\mathbf{x} \in \mathbb{C}^{N \times 1}$ has covariance matrix $\mathbb{E}\left[\mathbf{x x}^{*}\right]=\sigma_{x}^{2} \mathbf{I}_{N}$, where $\mathbb{E}[\cdot]$ stands for the expected value, $(\cdot)^{*}$ is the conjugate transpose, and $\mathbf{I}_{N}$ is an $N \times N$ identity matrix. The white Gaussian noise $\mathbf{z} \sim N\left(0, \sigma_{z}^{2} \mathbf{I}\right)$ is also circularly symmetric. The input signal-to-noise ratio (SNR) is defined as

$$
\operatorname{snr}=\frac{\sigma_{x}^{2}}{\sigma_{z}^{2}}
$$

In this paper, we present an in-depth analysis of the performance of the zero forcing (ZF) and minimum mean squared error (MMSE) equalizers applied to the channel given in (1). The linear ZF and MMSE equalizers are classic functional blocks and are ubiquitous in digital communications [1]. They are also the building blocks of more advanced communication schemes such as the decision feedback equalizer (DFE), or equivalently, the V-BLAST (vertical Bell Labs layered Space-Time) architecture [2][3], and various other MIMO transceiver designs (see, e.g., [4][5] and the references therein). Despite their fundamental importance, however, the existing performance analyses of the ZF and MMSE equalizers ${ }^{1}$ are far from complete. For instance, it is commonly understood that ZF is a limiting form of MMSE as snr $\rightarrow \infty$. But when the ZF and MMSE are applied to the MIMO fading channel given in (1), one may observe through simulations that the error probabilities of MMSE and ZF do not coincide even as snr $\rightarrow \infty$. To the best of our knowledge, no rigorous account of such a phenomenon is available in the literature. As another example, the problem of obtaining the exact diversity-multiplexing (D-M) tradeoff [6] of V-BLAST with optimal detection ordering still remains open, and so does the quantification of the gain due to optimal detection ordering. In this paper, we attempt to provide an in-depth look at the classical ZF and MMSE equalizers with respect to the well-known performance metrics of output SNR, uncoded error and outage probabilities, diversity-

\footnotetext{
${ }^{1}$ In the sequel we refer to the ZF and MMSE equalizers as ZF and MMSE for simplicity.
} 
multiplexing (D-M) gain tradeoff, and SNR gain.

The major findings of this paper are summarized in the following.

$\underline{R 1}$ A common perception about ZF and MMSE is that ZF is the limiting form of MMSE as snr $\rightarrow \infty$. Therefore, it is presumed that the two equalizers would share the same output SNRs, and consequently, the same uncoded error or outage probability in the high SNR regime. We show, however, that the output SNRs of the $N$ data substreams using MMSE and ZF are related by

$$
\rho_{\mathrm{mmse}, n}=\rho_{\mathrm{zf}, n}+\eta_{\mathrm{snr}, n}, \quad 1 \leq n \leq N,
$$

where $\rho_{\mathrm{zf}, n}$ and $\eta_{\mathrm{snr}, n}$ are statistically independent and $\eta_{\mathrm{snr}, n}$ is a nondecreasing function of snr. Moreover,

$$
\eta_{\mathrm{snr}, n} \rightarrow \eta_{\infty, n} \quad \text { with probability one (w.p.1), as snr } \rightarrow \infty
$$

where $\frac{M-N+2}{N-1} \eta_{\infty, n} \sim \mathcal{F}_{2(N-1), 2(M-N+2)}$ is of $\mathcal{F}$-distribution. ${ }^{2}$ Further, the interference-to-noise ratio (INR) of the $n$th substream at the output of MMSE (denoted as $\operatorname{inr}_{n}$ ), is approximately upper bounded as

$$
\operatorname{inr}_{n} \lesssim \frac{\eta_{\mathrm{snr}, n}}{\rho_{\mathrm{zf}, n}}
$$

with the approximate upper bound being asymptotically tight for high SNR. Since $\frac{\eta_{\mathrm{ssr}, n}}{\rho_{\mathrm{zf}, n}}$ is inversely proportional to the input SNR, (5) implies that the higher the input SNR, the smaller the leakage from the interfering substreams.

$\underline{R 2}$ Using $\underline{\mathrm{R} 1}$, we obtain tight approximations of the uncoded error and outage probabilities of MMSE which can be evaluated via numerical integration rather than Monte-Carlo simulations. This analysis also confirms that there is a non-vanishing SNR gain of MMSE over ZF as snr $\rightarrow \infty$. Interestingly, however, the $\epsilon$-outage capacities of MMSE and ZF coincide in the asymptotically high SNR regime in spite of the SNR gap between their outage probabilities.

$\underline{\text { R3 }}$ We obtain the following upper bounds of the output SNRs for the ZF and MMSE equalizers:

$$
\rho_{\mathrm{mmse}, n} \leq \frac{\lambda_{N}^{2} \mathrm{snr}+1}{u}-1 \quad \text { and } \quad \rho_{\mathrm{zf}, n} \leq \frac{\lambda_{N}^{2} \mathrm{snr}}{u},
$$

where $\lambda_{N}$ is the smallest singular value of $\mathbf{H}$ and $u$ is a Beta random variable that is independent of $\lambda_{N}$ with a probability density function (pdf)

$$
f_{u}(x)=(N-1)(1-x)^{N-2}, \quad 0 \leq x \leq 1 .
$$

${ }^{2}$ Given two independent Chi-square random variables $a \sim \chi_{m}^{2}$ and $b \sim \chi_{n}^{2}$. The ratio $c=\frac{a / m}{b / n}$ is a random variable with distribution $f_{c}(x)=\frac{\Gamma\left(\frac{m+n}{2}\right) n^{\frac{n}{2}} m^{\frac{m}{2}} x^{\frac{m}{2}-1}}{\Gamma\left(\frac{m}{2}\right) \Gamma\left(\frac{n}{2}\right)(n+m x)^{\frac{m+n}{2}}}$, where $\Gamma(z)=\int_{0}^{\infty} t^{z-1} e^{-t} d t$. We denote $c \sim \mathcal{F}_{m, n}[7]$. 
Based on these upper bounds, we prove that for both ZF and MMSE, the D-M gain tradeoff of a fictitious parallel channel (with $N$ independent sub-channels) with coding across the $N$ substreams is the same as that for the ZF and MMSE equalizers applied to the MIMO channel with independent coding over each individual substream, and this trade-off is given as

$$
d(r)=(M-N+1)\left(1-\frac{r}{N}\right)
$$

That is, the SNR gain gap between the MMSE and ZF equalizers cannot be captured by the D-M gain tradeoff analysis.

$\underline{R_{4}}$ As an important corollary of $\underline{\mathrm{R} 3}$, we solve the well-known open problem on the diversity gain of the V-BLAST architecture with optimal detection ordering [2]. Note that the V-BLAST architecture can be regarded as employing ZF or MMSE equalizers combined with decision feedback [3], which in the sequel are referred to simply as ZF-VB and MMSE-VB, respectively. We prove that with equal rate for each substream and for any order of decoding, both ZF-VB and MMSE-VB have the D-M gain tradeoff

$$
d_{\mathrm{vb}}(r)=(M-N+1)\left(1-\frac{r}{N}\right)
$$

which means that the so-called V-BLAST order [2] does not yield an improvement in the D-M gain tradeoff relative to unordered decoding.

$\underline{R} 5$ We also derive lower bounds on the outage probabilities of MIMO systems that use ZF and MMSE (without decision feedback). The lower bounds are shown to be asymptotically tight for high SNR. Based on these bounds, we prove that for ZF the strongest substream has a SNR gain of as much as $10 \log _{10} N$ dB over an average one at high SNR. For MMSE, the SNR gain is even higher, and that too by a significant margin. When applied to systems with decision feedback, as in V-BLAST, because the overall outage probability is dominated by that of the first detected substream, this result also quantifies the coding advantage of optimally ordered decoding over fixed order decoding.

The results $\underline{\mathrm{R} 1}$ and $\underline{\mathrm{R} 2}$ are on the distribution of the output SNR of the MMSE equalizer, the asymptotic normality of interference-plus-noise at its output, and the coded (outage) and uncoded error probability performance. Such problems are also investigated in [8][9] for the asymptotic property of linear multiuser receivers. While their work focuses on large systems, we study finite systems with asymptotically high SNR. The influence of non-Gaussian interference upon error probability in finite CDMA systems is studied in [10] which shows that the larger an interfering user's amplitude, the smaller its effect on bit-error rate [10]. The (tight) upper bound of INR given in (5) yields more 
insights into this observation. The output SINR decomposition (3) was proposed in the conference version of this paper [11]. In the independent work [12], the authors show that such a decomposition is possible even if the columns of $\mathbf{H}$ are correlated (but the rows need to be independent). In this case the pdf of the output SINR $\rho_{\mathrm{mmse}, n}$ is very involved. The approach of [12] is to approximate the first three asymptotic moments of $\eta_{\mathrm{snr}, n}$ as $M, N \rightarrow \infty$, and then approximate it by a Gamma (or generalized Gamma) random variable. Our strategy is to study the exact distribution of $\eta_{\mathrm{snr}, n}$ at asymptotically high SNR, which leads to a more concise approximation.

The results $\underline{\mathrm{R} 3}, \underline{\mathrm{R}} 4$, and $\underline{\mathrm{R} 5}$ are motivated by the problem of the D-M tradeoff of V-BLAST with ordered decoding. Although this problem has inspired much research, previous attempts have only achieved partial success and that too for the ZF-VB. For instance, it is shown in [13], [14] that optimal ordering does not improve the diversity gain of ZF-VB but that it provides a $3 \mathrm{~dB}$ SNR gain when there are two transmitting antennas $(N=2)$. The extension to the case of $N \leq 4$ can be found in [15]. It is also shown in [13] that a suboptimal column-norm ordering technique proposed in [16] does not improve the diversity gain for arbitrary $N$. Note that a (loose) upper bound to the D-M tradeoff of ZF-VB with optimal order detection is given in $[6]$ to be

$$
d_{\mathrm{vb}}(r) \leq(M-1)\left(1-\frac{r}{N}\right), \quad 0 \leq r \leq N
$$

The difficulty of this problem lies in the fact that the distribution of the layer gains becomes extremely complicated due to the channel-dependent detection ordering. We circumvent this difficulty by identifying the sharp upper bound given in (6). Indeed, the result $\underline{\mathrm{R} 5}$ is also related to the bound (6).

The rate/capacity performance of ZF and MMSE receivers applied to the point-to-point fading MIMO channel are addressed in [17], where the authors show that the average capacity loss due to using the linear ZF or MMSE equalizers converges to a constant as SNR increases. Similar conclusions with regard to the sum rate are made in [18] and [19] in the context of the multi-access channel (MAC) and the broadcast channel (BC). Combined with these results on rate/capacity performance, this paper provides a more detailed picture of the performance of ZF and MMSE applied to both single user and multiuser MIMO fading channels, especially in the high SNR regime.

The remainder of this paper is organized as follows. Section II introduces some preliminary results to be used in the paper. In Section III, we analyze the output SNR of MMSE. Section IV derives the uncoded error and outage probabilities of MMSE at high SNR. The D-M gain tradeoffs of the system 
using ZF and MMSE are derived in Section V. Based on a tight lower bound to the outage probabilities of the $N$ substreams, we derive the SNR gain of optimal detection ordering for V-BLAST in Section VI. Section VII presents the numerical examples validating the theoretical analysis. Conclusions are made in Section VIII.

\section{Preliminaries}

\section{A. Basics of ZF and MMSE Equalizers}

Consider the MIMO channel model given in (1) where the $N$ data substreams are mixed by the channel matrix. The ZF and MMSE equalizers can be applied to decouple the $N$ substreams. The ZF and MMSE equalization matrices are (see, e.g., [20])

$$
\mathbf{W}_{\mathrm{zf}}=\left(\mathbf{H}^{*} \mathbf{H}\right)^{-1} \mathbf{H}^{*}, \quad \text { and } \quad \mathbf{W}_{\mathrm{mmse}}=\left(\mathbf{H}^{*} \mathbf{H}+\frac{1}{\mathrm{snr}} \mathbf{I}\right)^{-1} \mathbf{H}^{*} .
$$

Left multiplying the received signal vector $\mathbf{y}$ by $\mathbf{W}_{\mathrm{zf}}$ and $\mathbf{W}_{\text {mmse }}$, we obtain $N$ decoupled substreams with output SNRs

$$
\rho_{\mathrm{zf}, n}=\frac{\mathrm{snr}}{\left[\left(\mathbf{H}^{*} \mathbf{H}\right)^{-1}\right]_{n n}}, \quad 1 \leq n \leq N
$$

and

$$
\rho_{\mathrm{mmse}, n}=\frac{\mathrm{snr}}{\left[\left(\mathbf{H}^{*} \mathbf{H}+\frac{1}{\mathrm{snr}} \mathbf{I}\right)^{-1}\right]_{n n}}-1, \quad 1 \leq n \leq N,
$$

respectively. Here $[\cdot]_{n n}$ denotes the $n$th diagonal element. Denote $\mathbf{h}_{n}$ the $n$th column of $\mathbf{H}$ and $\mathbf{H}_{n}$ the submatrix obtained by striking $\mathbf{h}_{n}$ out of $\mathbf{H}$. It follows from (12) and the fact (see, e.g., [21])

$$
\left[\left(\mathbf{H}^{*} \mathbf{H}\right)^{-1}\right]_{n n}=\frac{1}{\mathbf{h}_{n}^{*} \mathbf{h}_{n}-\mathbf{h}_{n}^{*} \mathbf{H}_{n}\left(\mathbf{H}_{n}^{*} \mathbf{H}_{n}\right)^{-1} \mathbf{H}_{n}^{*} \mathbf{h}_{n}}
$$

that

$$
\rho_{\mathrm{zf}, n}=\left[\mathbf{h}_{n}^{*} \mathbf{h}_{n}-\mathbf{h}_{n}^{*} \mathbf{H}_{n}\left(\mathbf{H}_{n}^{*} \mathbf{H}_{n}\right)^{-1} \mathbf{H}_{n}^{*} \mathbf{h}_{n}\right] \mathrm{snr}=\left(\mathbf{h}_{n}^{*} \mathbf{P}_{\mathbf{H}_{n}}^{\perp} \mathbf{h}_{n}\right) \mathrm{snr},
$$

where $\mathbf{P}_{\mathbf{H}_{n}}^{\perp}=\mathbf{I}-\mathbf{H}_{n}\left(\mathbf{H}_{n}^{*} \mathbf{H}_{n}\right)^{-1} \mathbf{H}_{n}^{*}$ stands for the orthogonal projection onto the null space of $\mathbf{H}_{n}^{*}$. In the case of i.i.d. Rayleigh fading, $\mathbf{h}_{n}^{*} \mathbf{P}_{\mathbf{H}_{n}}^{\perp} \mathbf{h}_{n} \sim \chi_{2(M-N+1)}^{2}$, with distribution [22]

$$
f_{\mathbf{h}_{n}^{*} \mathbf{P}_{\mathbf{H}_{n}}^{\perp} \mathbf{h}_{n}}(x)=\frac{1}{(M-N) !} x^{M-N} e^{-x}, \quad x \geq 0 .
$$

Similarly, we have an alternative expression for $\rho_{\mathrm{mmse}, n}[11]$ :

$$
\rho_{\text {mmse }, n}=\left[\mathbf{h}_{n}^{*} \mathbf{h}_{n}-\mathbf{h}_{n}^{*} \mathbf{H}_{n}\left(\mathbf{H}_{n}^{*} \mathbf{H}_{n}+\frac{1}{\mathrm{snr}} \mathbf{I}\right)^{-1} \mathbf{H}_{n}^{*} \mathbf{h}_{n}\right] \text { snr, } \quad 1 \leq n \leq N .
$$




\section{B. Diversity-Multiplexing Gain Tradeoff}

In [6], the authors established the framework of D-M gain tradeoff analysis in the asymptotically high SNR regime. Denote $R(\mathrm{snr})$ as the data rate of any communication scheme with input SNR snr. The diversity gain and multiplexing gain are defined as follows [6].

Definition II.1: A scheme is said to have multiplexing gain $r$ and diversity gain $d$ if the data rate $R($ snr) satisfies

$$
\lim _{\mathrm{snr} \rightarrow \infty} \frac{R(\mathrm{snr})}{\log \mathrm{snr}}=r,
$$

and the average error probability $P_{e}(\mathrm{snr})$ satisfies

$$
\lim _{\mathrm{snr} \rightarrow \infty} \frac{\log P_{e}(\mathrm{snr})}{\log \mathrm{snr}}=-d .
$$

Because $P_{e}(\mathrm{snr})$ and $R(\mathrm{snr})$ are related, so are $d$ and $r$. We denote $d(r)$ the tradeoff between the diversity gain and multiplexing gain, which is always a non-increasing function.

\section{Two Theorems}

The following two theorems turn out to be very useful for the analysis in this paper. The first theorem is a slight variation of [23, Lemma 2.6].

Theorem II.2: Let $\mathbf{H}$ be an $M \times N$ Gaussian matrix, whose entries are i.i.d. complex Gaussian random variables with zero-mean and unit variance. With $\mathbf{H}=\mathbf{U} \boldsymbol{\Lambda} \mathbf{V}^{*}$ being the singular value decomposition (SVD) of $\mathbf{H}$ we have that both $\mathbf{U}$ and $\mathbf{V}$ are Haar matrices ${ }^{3}$ and they are statistically independent of $\boldsymbol{\Lambda}$.

The second theorem is implied in [6].

Theorem II.3: For an $M \times N$ i.i.d. Rayleigh fading channel matrix $\mathbf{H}$ with ordered squared singular values of $\mathbf{H}, \lambda_{1}^{2} \geq \lambda_{2}^{2} \geq \cdots \geq \lambda_{N}^{2}>0$,

$$
\lim _{\epsilon \rightarrow 0_{+}} \frac{\log \mathbb{P}\left(\lambda_{n}^{2}<\epsilon\right)}{\log \epsilon}=(M-n+1)(N-n+1), \quad 1 \leq n \leq N .
$$

In other words,

$$
\mathbb{P}\left(\lambda_{n}^{2}<\epsilon\right)=\epsilon^{(M-n+1)(N-n+1)+o(1)}, \quad 1 \leq n \leq N .
$$

where $o(1)$ stands for a vanishing term as $\epsilon \rightarrow 0$.

\footnotetext{
${ }^{3} \mathrm{~A}$ random matrix is a Haar matrix if it is uniformly distributed on the set of unitary matrices.
} 


\section{Analysis of the Output SNR of MMSE}

Since the elements of the channel matrix $\mathbf{H}$ are i.i.d., the output SNRs of the $N$ substreams are of identical (but not independent) marginal distributions. Hence, to study the distribution of the output SNRs of the $N$ substreams, we only need to focus on one, say the $n$th substream. As shown in (15) and (16), $\rho_{\mathrm{zf}, n}$ is equal to snr multiplied by a Chi-square random variable. However, the distribution of the output SNR of MMSE is more complicated. We start with analyzing the gap between the output SNRs of ZF and MMSE.

It follows from (15) and (17) that the difference between $\rho_{\text {mmse }, n}$ and $\rho_{\mathrm{zf}, n}$, which we denote as $\eta_{\mathrm{snr}, n}$ is

$$
\eta_{\mathrm{snr}, n} \triangleq \rho_{\mathrm{mmse}, n}-\rho_{\mathrm{zf}, n}=\operatorname{snrh}_{n}^{*} \mathbf{H}_{n}\left[\left(\mathbf{H}_{n}^{*} \mathbf{H}_{n}\right)^{-1}-\left(\mathbf{H}_{n}^{*} \mathbf{H}_{n}+\frac{1}{\mathrm{snr}} \mathbf{I}\right)^{-1}\right] \mathbf{H}_{n}^{*} \mathbf{h}_{n}
$$

Since $\rho_{\mathrm{mmse}, n}=\rho_{\mathrm{zf}, n}+\eta_{\mathrm{snr}, n}$, the characterization of $\rho_{\mathrm{mmse}, n}$ is given by the following theorem.

Theorem III.1: The random variable $\eta_{\mathrm{snr}, n}$ is statistically independent of $\rho_{\mathrm{zf}, n}$. Moreover, as snr $\rightarrow$ $\infty$, it converges to a scaled $\mathcal{F}$ random variable w.p. 1. In particular,

$$
\eta_{\mathrm{snr}, n} \stackrel{\text { w.p.1 }}{\longrightarrow} \eta_{\infty, n}
$$

where

$$
\frac{M-N+2}{N-1} \eta_{\infty, n} \sim \mathcal{F}_{2(N-1), 2(M-N+2)} .
$$

Proof: Let $\mathbf{H}_{n}=\mathbf{U}_{n} \boldsymbol{\Lambda}_{n} \mathbf{V}_{n}^{*}$ be the SVD, where $\mathbf{U}_{n} \in \mathbb{C}^{M \times(N-1)}$ and $\boldsymbol{\Lambda}_{n} \in \mathbb{C}^{(N-1) \times(N-1)}$. Then

$$
\begin{aligned}
\eta_{\text {snr }, n} & =\operatorname{snrh}_{n}^{*} \mathbf{U}_{n} \boldsymbol{\Lambda}_{n}\left[\boldsymbol{\Lambda}_{n}^{-2}-\left(\boldsymbol{\Lambda}_{n}^{2}+\frac{1}{\mathrm{snr}} \mathbf{I}\right)^{-1}\right] \boldsymbol{\Lambda}_{n} \mathbf{U}_{n}^{*} \mathbf{h}_{n} \\
& =\mathbf{h}_{n}^{*} \mathbf{U}_{n}\left(\boldsymbol{\Lambda}_{n}^{2}+\frac{1}{\mathrm{snr}} \mathbf{I}\right)^{-1} \mathbf{U}_{n}^{*} \mathbf{h}_{n} .
\end{aligned}
$$

It is readily seen from (26) that given $\mathbf{H}_{n}$ and $\mathbf{h}_{n}, \eta_{\mathrm{snr}, n}$ is a non-decreasing function of snr. According to the i.i.d. Rayleigh fading assumption, $\mathbb{E}\left[\mathbf{h}_{n} \mathbf{h}_{n}^{*}\right]=\mathbf{I}$, thus

$$
\mathbb{E}\left[\mathbf{P}_{\mathbf{H}_{n}}^{\perp} \mathbf{h}_{n} \mathbf{h}_{n}^{*} \mathbf{U}_{n}\right]=\mathbb{E}_{\mathbf{H}_{n}}\left[\mathbb{E}_{\mathbf{h}_{n} \mid \mathbf{H}_{n}}\left[\mathbf{P}_{\mathbf{H}_{n}}^{\perp} \mathbf{h}_{n} \mathbf{h}_{n}^{*} \mathbf{U}_{n}\right]\right]=\mathbb{E}_{\mathbf{H}_{n}}\left[\mathbf{P}_{\mathbf{H}_{n}}^{\perp} \mathbf{U}_{n}^{*}\right]=0
$$

where the last equality follows by the fact that $\mathbf{P}_{\mathbf{H}_{n}}^{\perp} \mathbf{U}_{n}^{*}=0$ for any instantiation of $\mathbf{H}_{n}$. Since both $\mathbf{P}_{\mathbf{H}_{n}}^{\perp} \mathbf{h}_{n}$ and $\mathbf{U}_{n}^{*} \mathbf{h}_{n}$ are zero-mean Gaussian random vectors, (27) implies that $\mathbf{P}_{\mathbf{H}_{n}}^{\perp} \mathbf{h}_{n}$ is statistically independent of $\mathbf{U}_{n}^{*} \mathbf{h}_{n}$. Note that $\mathbf{P}_{\mathbf{H}_{n}}^{\perp} \mathbf{h}_{n}$ is also independent of $\boldsymbol{\Lambda}_{n}$, because $\mathbf{P}_{\mathbf{H}_{n}}^{\perp} \mathbf{h}_{n}=$ $\left(\mathbf{I}-\mathbf{U}_{n} \mathbf{U}_{n}^{*}\right) \mathbf{h}_{n}$ with both $\mathbf{h}_{n}$ and $\mathbf{U}_{n}$ independent of $\boldsymbol{\Lambda}_{n}$ (cf. Theorem II.2). Hence $\mathbf{P}_{\mathbf{H}_{n}}^{\perp} \mathbf{h}_{n}$ is independent of $\mathbf{h}_{n}^{*} \mathbf{U}_{n}\left(\boldsymbol{\Lambda}_{n}^{2}+\frac{1}{\operatorname{snr}} \mathbf{I}\right)^{-1} \mathbf{U}_{n}^{*} \mathbf{h}_{n}$, since the latter is a function of $\mathbf{U}_{n}^{*} \mathbf{h}_{n}$ and $\boldsymbol{\Lambda}_{n}$. Consequently, 
$\rho_{\mathrm{zf}, n}=\left\|\mathbf{P}_{\mathbf{H}_{n}}^{\perp} \mathbf{h}_{n}\right\|^{2}$ snr is also independent of $\eta_{\mathrm{snr}, n}=\mathbf{h}_{n}^{*} \mathbf{U}_{n}\left(\boldsymbol{\Lambda}_{n}^{2}+\frac{1}{\mathrm{snr}} \mathbf{I}\right)^{-1} \mathbf{U}_{n}^{*} \mathbf{h}_{n}$. Here $\|\cdot\|$ stands for the Euclidean norm of a vector.

It follows from (26) and the fact that the diagonal elements of $\boldsymbol{\Lambda}_{n}^{2}$ are all nonzero with probability one (w.p. 1) that

$$
\lim _{\mathrm{snr} \rightarrow \infty} \eta_{\mathrm{snr}, n}=\mathbf{h}_{n}^{*} \mathbf{U}_{n} \boldsymbol{\Lambda}_{n}^{-2} \mathbf{U}_{n}^{*} \mathbf{h}_{n}
$$

Defining $\eta_{\infty, n} \triangleq \mathbf{h}_{n}^{*} \mathbf{U}_{n} \boldsymbol{\Lambda}_{n}^{-2} \mathbf{U}_{n}^{*} \mathbf{h}_{n}$, we have shown that

$$
\eta_{\mathrm{snr}, n} \stackrel{\text { w.p.1 }}{\longrightarrow} \eta_{\infty, n}, \quad \text { as } \quad \text { snr } \rightarrow \infty .
$$

Because $\eta_{\mathrm{snr}, n}$ is independent of $\rho_{\mathrm{zf}, n}$, so is its limit $\eta_{\infty, n}$.

We now derive the distribution of $\eta_{\infty, n}$. Denoting $\mathbf{g}=\mathbf{U}_{n}^{*} \mathbf{h}_{n} \in \mathbb{C}^{(N-1) \times 1}$, we have that $\eta_{\infty, n}=$ $\mathbf{g}^{*} \boldsymbol{\Lambda}_{n}^{-2} \mathbf{g}$, where $\mathbf{g} \sim N(0, \mathbf{I})$ since $\mathbb{E}\left[\mathbf{g g}^{*}\right]=\mathbf{U}_{n}^{*} \mathbf{U}_{n}=\mathbf{I}_{N-1}$. Moreover, $\mathbf{g}$ and $\boldsymbol{\Lambda}_{n}$ are independent since the singular matrix and singular values are independent (cf. Theorem II.2). Consider a matrix $\mathbf{G} \in \mathbb{C}^{M \times(N-1)}$ which has the same dimension and distribution of $\mathbf{H}_{n}$ and is independent of $\mathbf{g}$. Using the SVD of $\mathbf{G}=\mathbf{U}_{G} \boldsymbol{\Lambda}_{G} \mathbf{V}_{G}^{*}$, we have $\mathbf{g}^{*}\left(\mathbf{G}^{*} \mathbf{G}_{n}\right)^{-1} \mathbf{g}=\mathbf{g}^{*} \mathbf{V}_{G} \boldsymbol{\Lambda}_{G}^{-2} \mathbf{V}_{G}^{*} \mathbf{g}$. It is seen that ${ }^{4}$

$$
\mathbf{V}_{G}^{*} \mathbf{g} \sim \mathbf{g}, \quad \mathbf{\Lambda}_{G} \sim \boldsymbol{\Lambda}_{n}
$$

and $\mathbf{V}_{G}^{*} \mathbf{g}$ is independent of $\boldsymbol{\Lambda}_{G}$. Consequently, we have

$$
\eta_{\infty, n} \sim \mathbf{g}^{*}\left(\mathbf{G}^{*} \mathbf{G}\right)^{-1} \mathbf{g}
$$

Construct a unitary matrix $\mathbf{U}_{g}$ such that $\mathbf{U}_{g} \mathbf{g}=\left[\mathbf{0}^{T},\|\mathbf{g}\|\right]^{T}$. Note that $\mathbf{U}_{g}$ is hence a Householder matrix [24]. Then

$$
\eta_{\infty, n}=\left[\mathbf{0}^{T},\|\mathbf{g}\|\right]\left(\mathbf{U}_{g} \mathbf{G}^{*} \mathbf{G} \mathbf{U}_{g}^{*}\right)^{-1}\left[\mathbf{0}^{T},\|\mathbf{g}\|\right]^{T} .
$$

Observe that $\mathbf{G}^{*} \mathbf{G}$ is statistically invariant under unitary transformations. Hence,

$$
\eta_{\infty, n} \sim\left[\mathbf{0}^{T},\|\mathbf{g}\|\right]\left(\mathbf{G}^{*} \mathbf{G}\right)^{-1}\left[\mathbf{0}^{T},\|\mathbf{g}\|\right]^{T}=\|\mathbf{g}\|^{2}\left[\left(\mathbf{G}^{*} \mathbf{G}\right)^{-1}\right]_{(N-1),(N-1)}
$$

It is clear that $\|\mathbf{g}\|^{2}$ is a Chi-Square random variable with $2(N-1)$ degrees of freedom, i.e., $\|\mathbf{g}\|^{2} \sim$ $\chi_{2(N-1)}^{2}$. According to (14) and (16),

$$
\frac{1}{\left[\left(\mathbf{G}^{*} \mathbf{G}\right)^{-1}\right]_{(N-1),(N-1)}} \sim \chi_{2(M-N+2)}^{2} .
$$

\footnotetext{
${ }^{4}$ By $a \sim b$, we mean that the random variables $a$ and $b$ have identical distribution.
} 
Hence, we have

$$
\eta_{\infty, n} \sim \frac{X}{Y}
$$

where $X \sim \chi_{2(N-1)}^{2}$ and $Y \sim \chi_{2(M-N+2)}^{2}$, or equivalently that

$$
\frac{M-N+2}{N-1} \eta_{\infty, n} \sim \mathcal{F}_{2(N-1), 2(M-N+2)},
$$

with the pdf of $\eta_{\infty, n}$ given as

$$
f_{\eta_{\infty, n}}(x)=\frac{M !}{(N-2) !(M-N+1) !} \frac{x^{N-2}}{(1+x)^{M+1}}, \quad 0 \leq x<\infty .
$$

Intuitively, $\eta_{\infty, n}$ represents the power of the signal component "hiding" in the range space of $\mathbf{H}_{n}$ that is recovered by the MMSE equalizer. In contrast, the ZF equalizer nulls out that signal component.

For any full rank channel matrix, $\frac{\eta_{\mathrm{snr}, n}}{\rho_{\mathrm{zf}, n}} \rightarrow 0$ as snr $\rightarrow \infty$. Therefore, the interference from the other data substreams is negligible compared to the channel noise as snr $\rightarrow \infty$. Consequently, for any full rank channel realization, the ratio of the output SNR gains (in $\mathrm{dB}$ ) of the MMSE to ZF equalizers goes to unity or

$$
10 \log _{10} \frac{\rho_{\mathrm{mmse}, n}}{\rho_{\mathrm{zf}, n}}=10 \log _{10}\left(1+\frac{\eta_{\mathrm{snr}, n}}{\rho_{\mathrm{zf}, n}}\right) \rightarrow 0, \quad \text { as snr } \rightarrow \infty
$$

In spite of the diminishing relative output SNR gain, the MMSE is shown to have remarkable SNR gain over ZF even as snr $\rightarrow \infty$ owing to the fact that the limit of their difference is an $\mathcal{F}$ random variable.

In the next section, we will provide applications of our analysis of $\rho_{\mathrm{mmse}, n}=\rho_{\mathrm{zf}, n}+\eta_{\mathrm{snr}, n}$. It is noted here that Theorem III.1 was originally presented in the conference version of this paper [11]. In the independent work of [12], the authors show that $\rho_{\mathrm{zf}, n}$ and $\eta_{\mathrm{snr}, n}$ are independent even if the columns of $\mathbf{H}$ are correlated but with the rows of $\mathbf{H}$ being independent. However, in this case, the exact distribution of $\eta_{\infty, n}$ is unknown.

\section{A. Interference-to-noise ratio (INR)}

In recovering the signal $x_{n}$ in the range space of $\mathbf{H}_{n}$, the MMSE equalizer admits some leakage from the other interfering data substreams. It is shown in [10] that the leakage diminishes as input power increases. A more careful study detailed in Appendix A shows that the INR at the output of the MMSE equalizer is in fact inversely proportional to the input SNR. 
Lemma III.2: The INR of the $n$th substream obtained using MMSE equalizer is upper bounded by

$$
\operatorname{inr}_{n} \lesssim \frac{\eta_{\mathrm{snr}, n}}{\rho_{\mathrm{zf}, n}}
$$

This upper bound is asymptotically tight at high SNR.

Proof: See Appendix A.

\section{Applications of Theorem III.1}

In this section, we apply Theorem III.1, i.e. the relationship $\rho_{\text {mmse }}=\rho_{\mathrm{zf}, n}+\eta_{\mathrm{snr}}$, to analyze the uncoded error probability, outage probability, and $\epsilon$-outage capacity of the MMSE equalizer. We shall see that the gap $\eta_{\text {snr }}$ brings about a remarkable difference in performance between the MMSE and ZF with respect to the uncoded error probability and outage probability as snr $\rightarrow \infty$. Interestingly however, their $\epsilon$-outage capacities coincide in the high SNR regime because this performance metric depends only on the fact that the ratio of the output SNRs of the MMSE and ZF equalizers approaches unity with increasing SNR.

\section{A. Uncoded Error Probability Analysis}

The uncoded error probability of the ZF equalizer is well known but we state it here for the sake of completeness. Consider the input of binary phase-shift keying (BPSK). The error probability of the $n$th substream obtained by ZF is (cf. (16))

$$
P_{b, \mathrm{zf}}=\int_{0}^{\infty} Q(\sqrt{2 \mathrm{snr} x}) \frac{1}{(M-N) !} x^{M-N} e^{-x} d x,
$$

where the $Q$-function is $Q(x)=\frac{1}{\sqrt{2 \pi}} \int_{x}^{\infty} e^{-\frac{t^{2}}{2}} d t$. The exact closed-form expression of $P_{b, \mathrm{zf}}$ is known (see, e.g., [20]).

$$
P_{b, \mathrm{zf}}=\left[\frac{1}{2}\left(1-\sqrt{\frac{\mathrm{snr}}{1+\mathrm{snr}}}\right)\right]^{M-N+1} \sum_{n=0}^{M-N+1}\left(\begin{array}{c}
M-N+n \\
n
\end{array}\right)\left(\frac{1+\sqrt{\frac{\mathrm{snr}}{1+\mathrm{snr}}}}{2}\right) .
$$

We now consider the problem of analyzing the uncoded error probability for the MMSE equalizer. Because the output SNRs of all the $N$ substreams are of identical distribution, we only need to focus on one substream.

For the error probability of MMSE, we assume that the sum of the perturbations due to the interference from the other data substreams and the channel noise can be well approximated as being Gaussian. Consequently, the approximate error probability of MMSE equalizer can be calculated 
through the $Q$-function (this Gaussian approximation is remarked on later):

$$
P_{b, \mathrm{mmse}} \simeq \mathbb{E}_{\rho_{\mathrm{mmse}, n}}\left[Q\left(\sqrt{2 \rho_{\mathrm{mmse}, n}}\right)\right] .
$$

It follows from (22) and (29) that

$$
\rho_{\mathrm{mmse}, n} \stackrel{w \cdot p .1}{\longrightarrow} \rho_{\mathrm{zf}, n}+\eta_{\infty, n}, \quad \text { as } \quad \mathrm{snr} \rightarrow \infty .
$$

Applying the Taylor expansion to $Q\left(\sqrt{2 \rho_{\mathrm{mmse}, n}}\right)$ around $\rho_{\mathrm{zf}, n}+\eta_{\infty, n}$, we obtain

$$
Q\left(\sqrt{2 \rho_{\mathrm{mmse}, n}}\right)=Q\left(\sqrt{2\left(\rho_{\mathrm{zf}, n}+\eta_{\infty, n}\right)}\right)+\frac{\Phi(\sqrt{2 \xi})}{\sqrt{2 \xi}}\left(\eta_{\infty, n}-\eta_{\mathrm{snr}, n}\right),
$$

where $\xi \in\left(\rho_{\text {mmse }, n}, \rho_{\mathrm{zf}, n}+\eta_{\infty, n}\right)$ and $\Phi(x)=\frac{1}{\sqrt{2 \pi}} \exp \left(-\frac{x^{2}}{2}\right)$. Recall that [25]

$$
\left(1-\frac{1}{x^{2}}\right) \frac{\Phi(x)}{x} \leq Q(x) \leq \frac{\Phi(x)}{x} .
$$

Therefore $\frac{\Phi(\sqrt{2 \xi})}{\sqrt{2 \xi}} \approx Q(\sqrt{2 \xi})$ at high SNR. Also note that $\xi \rightarrow \rho_{\mathrm{zf}, n}+\eta_{\infty, n}$ and $\eta_{\infty, n}-\eta_{\mathrm{snr}, n} \rightarrow 0$ w.p.1 as snr $\rightarrow \infty$. We can see from (42) that

$$
Q\left(\sqrt{2 \rho_{\mathrm{mmse}, n}}\right)=Q\left(\sqrt{2\left(\rho_{\mathrm{zf}, n}+\eta_{\infty, n}\right)}\right)(1+o(1)) \quad \text { w.p. } 1 .
$$

Hence, at high SNR, we have that

$$
\lim _{\mathrm{snr} \rightarrow \infty} \frac{\mathbb{E}\left[Q\left(\sqrt{2 \rho_{\mathrm{mmse}, n}}\right)\right]}{\mathbb{E}\left[Q\left(\sqrt{2\left(\rho_{\mathrm{zf}, n}+\eta_{\infty, n}\right)}\right)\right]}=1,
$$

so that the error probability of MMSE can be further approximated as

$$
P_{b, \mathrm{mmse}} \simeq \mathbb{E}\left[Q\left(\sqrt{2\left(\rho_{\mathrm{zf}, n}+\eta_{\infty, n}\right)}\right)\right] .
$$

Since the distributions of $\rho_{\mathrm{zf}, n}$ and $\eta_{\infty, n}$ are given in (16) and (36), respectively, (46) can be obtained via numerical integration rather than Monte Carlo simulations. Invoking the alternative expression of $Q(x)[26]$, namely that

$$
Q(x)=\frac{1}{\pi} \int_{0}^{\pi / 2} \exp \left(-\frac{x^{2}}{2 \sin ^{2} \theta}\right) d \theta
$$

we have

$$
\begin{aligned}
P_{b, \mathrm{mmse}} & \simeq E_{\eta_{\infty, n}}\left[\frac{1}{\pi} \int_{0}^{\infty} \int_{0}^{\pi / 2} \exp \left(-\frac{2\left(\operatorname{snr} x+\eta_{\infty, n}\right)}{2 \sin ^{2} \theta}\right) d \theta \frac{1}{(M-N) !} x^{M-N} e^{-x} d x\right] \\
& <\mathbb{E}_{\eta_{\infty, n}}\left[e^{-\eta_{\infty, n}} \frac{1}{\pi} \int_{0}^{\infty} \int_{0}^{\pi / 2} \exp \left(-\frac{\operatorname{snr} x}{\sin ^{2} \theta}\right) d \theta \frac{1}{(M-N) !} x^{M-N} e^{-x} d x\right] \\
& =\mathbb{E}\left[e^{-\eta_{\infty, n}}\right] P_{b, \mathrm{zf}}
\end{aligned}
$$


where to obtain (49), we have used the fact that $e^{-\eta_{\infty, n}} \geq e^{-\frac{\eta_{\infty, n}}{\sin ^{2} \theta}}$. Note that $\mathbb{E}\left[e^{-\eta_{\infty, n}}\right]$ is a constant number strictly less than unity. The equation (50) shows the non-vanishing error probability gap between ZF and MMSE even in the high SNR regime.

Calculating the error probabilities of a general quadrature amplitude modulation (QAM) is straightforward using the error probability expression in Q-function [27].

The representation of the error probability using the $Q$-function is based on the Gaussian approximation of the perturbation due to interference-plus-noise, and hence is not exact. In [10], Poor, et. al. show that in some scenarios the error probability calculated based on the Gaussian approximation is indiscernible from the exact one. It is explained essentially by observing that (i) the leakage from the interfering substreams diminishes at high SNR and (ii) the interference term is dominated by the noise at low SNR. In either case, the perturbation can be well-approximated by Gaussian noise. But their work focused on non-fading channel. In fading channels, however, a rigorous justification for the Gaussian approximation is still missing. Indeed, we have observed through extensive simulations that for rank-deficient channel realizations there is a non-negligible discrepancy between the $Q$-function approximation and the actual one, especially for channels with low dimensionality (say, $M=N=2$ ). Despite this phenomenon, the Gaussian approximation is still quite accurate in terms of average error probability for channels that are full rank with probability one, a fact that is verified in a numerical example given in Section VII.

\section{B. Outage Probability and $\epsilon$-Outage Capacity}

Consider employing independent codes of rate $R$ each over the $N$ antennas. The $n$th antenna transmission is in outage if the output SNR cannot support the target rate $R$. With the ZF equalizer the outage probability of the $n$th substream is

$$
P_{\mathrm{out}, n}^{\mathrm{zf}}=\mathbb{P}\left(\log \left(1+\rho_{\mathrm{zf}, n}\right)<R\right)=F_{\chi_{2(M-N+1)}^{2}}\left(\frac{2^{R}-1}{\mathrm{snr}}\right)
$$


where $F_{\chi_{2(M-N+1)}^{2}}(x)=1-e^{-x} \sum_{k=0}^{M-N} \frac{x^{k}}{k !}$ is the cumulative density function (cdf) of $\chi_{2(M-N+1)}^{2}$. The outage probability of MMSE is

$$
\begin{aligned}
P_{\mathrm{out}, n}^{\mathrm{mmse}} & =\mathbb{P}\left(\log \left(1+\rho_{\mathrm{zf}, n}+\eta_{\mathrm{snr}, n}\right)<R\right) \\
& =\int_{0}^{2^{R}-1} F_{\chi_{2(M-N+1)}^{2}}\left(\frac{2^{R}-1-\eta}{\mathrm{snr}}\right) f_{\eta_{\mathrm{snr}, n}}(\eta) d \eta \\
& \simeq \int_{0}^{2^{R}-1} F_{\chi_{2(M-N+1)}^{2}}\left(\frac{2^{R}-1-\eta}{\mathrm{snr}}\right) f_{\eta_{\infty, n}}(\eta) d \eta .
\end{aligned}
$$

We impose the upper limit $2^{R}-1$ on the integration because $F_{\chi_{2(M-N+1)}^{2}}(x)=0$ when $x<0$. Inserting (36) into (52), we calculate the outage probability of MMSE using numerical integration. Note that

$$
F_{\chi_{2(M-N+1)}^{2}}(x)=e^{-x} \sum_{k=M-N+1}^{\infty} \frac{x^{k}}{k !}=\frac{x^{(M-N+1)}}{(M-N+1) !}+o\left(x^{(M-N+1)}\right)
$$

around the origin. It follows from (52) that for high SNR (snr $\left.\gg 2^{R}-1\right)$,

$$
\begin{aligned}
P_{\mathrm{out}, n}^{\mathrm{mmse}} & \approx F_{\chi_{2(M-N+1)}^{2}}\left(\frac{2^{R}-1}{\mathrm{snr}}\right) \int_{0}^{2^{R}-1}\left(1-\frac{\eta}{2^{R}-1}\right)^{M-N+1} f_{\eta_{\infty, n}}(\eta) d \eta \\
& =P_{\mathrm{out}, n}^{\mathrm{zf}} \int_{0}^{2^{R}-1}\left(1-\frac{\eta}{2^{R}-1}\right)^{M-N+1} f_{\eta_{\infty, n}}(\eta) d \eta
\end{aligned}
$$

Given a fixed rate $R$, there is a non-vanishing gap between $P_{\mathrm{out}, n}^{\mathrm{mmse}}$ and $P_{\mathrm{out}, n}^{\mathrm{zf}}$ even as snr $\rightarrow \infty$. Moreover, as we can observe from (53), the gap would become smaller as $R$ increases. This phenomenon is validated in Section VII on numerical results.

The $\epsilon$-outage capacity is the maximum supportable rate under the restriction that the outage probability is no greater than $\epsilon$, and is defined as

$$
C_{\mathrm{zf}}(\epsilon) \triangleq \sup \left\{R: \mathbb{P}\left(\log \left(1+\rho_{\mathrm{zf}, n}\right)<R\right) \leq \epsilon\right\}
$$

and

$$
\begin{aligned}
C_{\mathrm{mmse}}(\epsilon) & \triangleq \sup \left\{R: \mathbb{P}\left(\log \left(1+\rho_{\mathrm{mmse}, n}\right)<R\right) \leq \epsilon\right\} \\
& =\sup \left\{R: \mathbb{P}\left(\log \left(1+\rho_{\mathrm{zf}, n}+\eta_{\mathrm{snr}, n}\right)<R\right) \leq \epsilon\right\}
\end{aligned}
$$

respectively. Since the cdfs of both $\rho_{\mathrm{zf}, n}$ and $\rho_{\text {mmse }, n}$ are continuous. It is easy to show that the cdfs of $\log \left(1+\rho_{\mathrm{zf}, n}\right)$ and $\log \left(1+\rho_{\mathrm{mmse}, n}\right)$ are also continuous. Therefore $C_{\mathrm{zf}}$ and $C_{\mathrm{mmse}}$ are the solutions to $\mathbb{P}\left(\log \left(1+\rho_{\mathrm{zf}, n}\right)<R\right)=\epsilon$ and $\mathbb{P}\left(\log \left(1+\rho_{\mathrm{mmse}, n}\right)<R\right)=\epsilon$, respectively. Now we obtain that

$$
C_{\mathrm{zf}}(\epsilon)=\log \left(1+\operatorname{snr} F_{\chi_{2(M-N+1)}^{2}}^{-1}(\epsilon)\right),
$$


where $F_{\chi_{2(M-N+1)}^{2}}^{-1}$ is the inverse function of the cdf of $\chi_{2(M-N+1)}^{2}$ thus satisfying

$$
\int_{0}^{F^{-1} \chi_{2(M-N+1)}^{2}}{ }^{(\epsilon)} \frac{1}{(M-N) !} x^{M-N} e^{-x} d x=\epsilon .
$$

For $C_{\mathrm{mmse}}(\epsilon)$, we examine the relationship:

$$
\begin{aligned}
& \mathbb{P}\left(\log \left(1+\rho_{\mathrm{mmse}, n}\right)<C_{\mathrm{zf}}(\epsilon)\right) \\
= & \mathbb{P}\left(\rho_{\mathrm{zf}, n}+\eta_{\mathrm{snr}, n}<\operatorname{snr} F_{\chi_{2(M-N+1)}^{2}}^{-1}(\epsilon)\right) \\
= & \mathbb{P}\left(\chi_{2(M-N+1)}^{2}<F_{\chi_{2(M-N+1)}^{2}}^{-1}(\epsilon)-\frac{\eta_{\mathrm{snr}, n}}{\mathrm{snr}}\right)
\end{aligned}
$$

By the continuity of the cdf,

$$
\lim _{\mathrm{snr} \rightarrow \infty} \mathbb{P}\left(\chi_{2(M-N+1)}^{2}<F_{\chi_{2(M-N+1)}^{2}}^{-1}(\epsilon)-\frac{\eta_{\mathrm{snr}, n}}{\mathrm{snr}}\right)=\mathbb{P}\left(\chi_{2(M-N+1)}^{2}<F_{\chi_{2(M-N+1)}^{2}}^{-1}(\epsilon)\right)=\epsilon, \quad \text { w.p.1. }
$$

Hence we have that $\lim _{\mathrm{snr} \rightarrow \infty} \mathbb{P}\left(\log \left(1+\rho_{\mathrm{mmse}, n}\right)<C_{\mathrm{zf}}(\epsilon)\right)=\epsilon$ w.p. 1 , and consequently,

$$
\lim _{\mathrm{snr} \rightarrow \infty} C_{\mathrm{mmse}}(\epsilon)-C_{\mathrm{zf}}(\epsilon)=0, \quad \text { w.p.1, }
$$

i.e., the MMSE equalizer has the same $\epsilon$-outage capacity as ZF at asymptotically high SNR.

The non-vanishing SNR gap between the outage probabilities of the zero-forcing and MMSE equalizers and the result in (59) may seem contradictory at first. The explanation for this apparent contradiction is that the difference between the outage probabilities of the two equalizers vanishes as the rate increases such that the difference between the maximum rates achievable with ZF and MMSE such that outage probability is less than a fixed threshold shrinks to zero with increasing SNR, a phenomenon we illustrate again in the section on numerical results.

\section{Analysis of D-M Gain Tradeoff}

In this section, we obtain the exact D-M gain tradeoffs of the linear ZF and MMSE receivers when independent, equal rate (and equal power) SISO Gaussian codebooks are employed over the $N$ antennas. As a by-product of this analysis, we further infer that no channel dependent ordering of substream decoding can improve the D-M tradeoff of V-BLAST.

\section{A. The Linear ZF Equalizer}

Consider the MIMO system that employs independent coding for each substream and the ZF equalizer at the receiver. Each substream effectively experiences a scalar channel whose gain is of $\chi_{2(M-N+1)}^{2}$ 
distribution. With the overall multiplexing gain $r$, each substream has a multiplexing gain $\frac{r}{N}$. The system is in outage if and only if at least one substream is in outage. Hence, the overall system outage probability is given as

$$
P_{\text {out }, \mathrm{zf}}(\mathrm{snr})=\mathbb{P}\left(\log \left(1+\frac{\mathrm{snr}}{\left[\left(\mathbf{H}^{*} \mathbf{H}\right)^{-1}\right]_{n n}}\right)<\frac{r}{N} \log \text { snr, for some } n\right),
$$

where $\mathbb{P}(\mathcal{E})$ denotes the probability of event $\mathcal{E}$. We can bound $(60)$ by

$\mathbb{P}\left(\log \left(1+\frac{\text { snr }}{\left[\left(\mathbf{H}^{*} \mathbf{H}\right)^{-1}\right]_{11}}\right)<\frac{r}{N} \log\right.$ snr $) \leq P_{\text {out }, \mathrm{zf}}(\mathrm{snr}) \leq \sum_{n=1}^{N} \mathbb{P}\left(\log \left(1+\frac{\mathrm{snr}}{\left[\left(\mathbf{H}^{*} \mathbf{H}\right)^{-1}\right]_{n n}}\right)<\frac{r}{N} \log\right.$ snr $)$.

Because the output SNRs of the $N$ substreams have identical distributions, the rightmost expression of $(61)$ is

$$
N \cdot \mathbb{P}\left(\log \left(1+\frac{\text { snr }}{\left[\left(\mathbf{H}^{*} \mathbf{H}\right)^{-1}\right]_{11}}\right)<r \log \mathrm{snr}\right) .
$$

Since $\frac{\log N}{\log \mathrm{snr}} \stackrel{\mathrm{snr} \rightarrow \infty}{\longrightarrow} 0$, according to (19), the diversity gain

$$
\begin{aligned}
d_{\mathrm{zf}}(r)=-\lim _{\mathrm{snr} \rightarrow \infty} \frac{\log P_{\text {out, } \mathrm{zf}}(\mathrm{snr})}{\log \mathrm{snr}} & =-\lim _{\mathrm{snr} \rightarrow \infty} \frac{\log \mathbb{P}\left(\log \left(1+\frac{\mathrm{snr}}{\left[\left(\mathbf{H}^{*} \mathbf{H}\right)^{-1}\right]_{11}}\right)<\frac{r}{N} \log \mathrm{snr}\right)}{\log \mathrm{snr}} \\
& =-\lim _{\mathrm{snr} \rightarrow \infty} \frac{\log \mathbb{P}\left(\frac{1}{\left[\left(\mathbf{H}^{*} \mathbf{H}\right)^{-1}\right]_{11}}<\operatorname{snr} \frac{r}{N}-1\right)}{\log \mathrm{snr}}
\end{aligned}
$$

Using the fact that $\frac{1}{\left[\left(\mathbf{H}^{*} \mathbf{H}\right)^{-1}\right]_{11}}$ has pdf given in (16), one can easily derive from (64) that (see [20])

$$
d_{\mathrm{zf}}(r)=(M-N+1)\left(1-\frac{r}{N}\right) .
$$

\section{B. The Linear MMSE Equalizer}

Analogously to (60), the outage probability is given as

$$
P_{\text {out,mmse }}(\text { snr })=\mathbb{P}\left(\log \left(\frac{\text { snr }}{\left[\left(\mathbf{H}^{*} \mathbf{H}+\frac{1}{\text { snr }} \mathbf{I}\right)^{-1}\right]_{n n}}\right)<\frac{r}{N} \log \text { snr, for some } n\right),
$$

Since the MMSE equalizer has a higher output SNR than the ZF equalizer (by $\eta_{s n r, n}$, a non-negative random variable), we have that $P_{\text {out,mmse }} \leq P_{\text {out,zf }}$. Therefore, it must be true that the D-M gain tradeoff for MMSE is better than that for ZF, i.e.,

$$
d_{\mathrm{mmse}}(r) \triangleq-\lim _{\mathrm{snr} \rightarrow \infty} \frac{\log P_{\mathrm{out}, \mathrm{mmse}}(\mathrm{snr})}{\log \mathrm{snr}} \geq(M-N+1)\left(1-\frac{r}{N}\right)
$$

Interestingly, equality in the above inequality holds, as we shall see next. 
We first prove the following lemma on the distribution of the elements of a column of a Haar matrix and their minimum value.

Lemma V.1: The joint pdf of $\left\{\left|v_{n}\right|^{2}\right\}_{n=1}^{N-1}$, where $\mathbf{v} \triangleq\left[v_{1} v_{2} \cdots v_{N}\right]^{T}$ is a column vector of a Haar matrix $\mathbf{V} \in \mathbb{C}^{N \times N}$, is ${ }^{5}$

$$
f_{\left|v_{1}\right|^{2}, \cdots,\left|v_{N-1}\right|^{2}}\left(x_{1}, \ldots, x_{N-1}\right)=(N-1) !, \quad \text { for } 0 \leq\left|x_{n}\right|^{2} \leq 1 \text { and } 0 \leq \sum_{n=1}^{N-1}\left|v_{n}\right|^{2} \leq 1 .
$$

The marginal pdf of $\left|v_{n}\right|^{2}$ for each $n$ is

$$
f_{\left|v_{n}\right|^{2}}(x)=(N-1)(1-x)^{N-2}, \quad 0 \leq x \leq 1,
$$

Define $u \triangleq \min \left\{\left|v_{n}\right|^{2}, n=1, \cdots, N.\right\}$. Then $u$ has pdf

$$
f_{u}(x)=\left(N^{2}-N\right)(1-N x)^{N-2}, \quad 0 \leq x \leq \frac{1}{N} .
$$

Consequently, for any fixed $n,\left|v_{n}\right|^{2}$ has the same distribution as $N u$, i.e., $\left|v_{n}\right|^{2} \sim N u$.

Proof: As $\mathbf{v}$ is a column of a Haar matrix,

$$
\mathbf{v} \sim \frac{\zeta}{\|\zeta\|}
$$

where $\boldsymbol{\zeta} \sim N\left(0, \mathbf{I}_{N}\right)$ is a complex-valued circularly symmetric Gaussian vector. Denote $X_{i} \triangleq\left|\zeta_{i}\right|^{2}$. Then $X_{i}$ 's are i.i.d. with an exponential distribution, i.e., $f_{X_{i}}(x)=e^{-x}$ for $x \geq 0$. Consider the conditional joint distribution of $X_{i}$ for $1 \leq i \leq N-1$ given $Y \triangleq\|\boldsymbol{\zeta}\|^{2}$. Using the fact that $Y=$ $\sum_{i=1}^{N} X_{i} \sim \chi_{2 N}^{2}$, we obtain

$$
\begin{aligned}
f_{X_{1}, \ldots, X_{N-1} \mid Y}\left(x_{1}, \ldots, x_{N-1} \mid y\right) & =\frac{f_{Y \mid X_{1}, \ldots, X_{N-1}}\left(y \mid x_{1}, \ldots, x_{N-1}\right) f\left(x_{1}, \ldots, x_{N-1}\right)}{f_{Y}(y)} \\
& =\frac{e^{-\sum_{i=1}^{N} x_{i}}}{\frac{1}{(N-1) !} y^{N-1} e^{-y}}=(N-1) ! y^{-(N-1)} .
\end{aligned}
$$

As $\left|v_{i}\right|=\frac{\left|\zeta_{i}\right|^{2}}{\|\boldsymbol{\zeta}\|^{2}}=\frac{X_{i}}{Y}$, it follows from (71) that

$$
f_{\left|v_{1}\right|^{2}, \ldots,\left|v_{N-1}\right|^{2} \mid Y}\left(x_{1}, \ldots, x_{N-1} \mid y\right)=(N-1) !
$$

Since the joint pdf of $\left\{\left|v_{n}\right|^{2}\right\}_{n=1}^{N-1}$ is independent of $Y$, we have proven (68). Note that the random vector $\left[\left|v_{1}\right|^{2}, \ldots,\left|v_{N-1}\right|^{2}\right]$ has a uniform distribution over the simplex

$$
\mathcal{S}=\left\{\left\{\left|v_{n}\right|^{2}\right\}_{n=1}^{N-1}: 0 \leq\left|v_{n}\right|^{2} \leq 1, \sum_{n=1}^{N-1}\left|v_{n}\right|^{2} \leq 1\right\}
$$

${ }^{5}$ Here we present the joint pdf of $\left\{\left|v_{n}\right|^{2}\right\}_{n=1}^{N-1}$ rather than $\left\{\left|v_{n}\right|^{2}\right\}_{n=1}^{N}$ because the latter is a degenerated function since $\sum_{i=1}^{N}\left|v_{i}\right|^{2}=1$. 
which has volume $\operatorname{Vol}(\mathcal{S})=\frac{1}{(N-1) !}$.

By the property of symmetry, $\left|v_{n}\right|^{2}$ 's have identical distribution. Note that $\left|v_{n}\right|^{2}$ has the same distribution as $\frac{Z_{1}}{Z_{1}+Z_{2}}$ where the two independent Chi-square random variables $Z_{1} \sim \chi_{2}^{2}$ and $Z_{2} \sim$ $\chi_{2 N-2}^{2}$. Hence $\left|v_{n}\right|^{2}$ is of Beta distribution with parameter $(1, N-1)$ (see, e.g., [28, pp. 60]):

$$
f_{\left|v_{n}\right|^{2}}(x)=(N-1)(1-x)^{N-2}, \quad 0 \leq x \leq 1
$$

According to the definition $u \triangleq \min \left\{\left|v_{n}\right|^{2}, n=1, \cdots, N\right.$. $\}$, we have

$$
\mathbb{P}(u>x)=\mathbb{P}\left(\left|v_{n}\right|^{2}>x, 1 \leq n \leq N\right) .
$$

It is not difficult to verify that the set $\left\{\left|v_{n}\right|^{2}>x, 1 \leq n \leq N\right\}$ corresponds to a subset of $\mathcal{S}$ :

$$
\left\{\left\{\left|v_{n}\right|^{2}\right\}_{n=1}^{N-1}:\left|v_{n}\right|^{2}>x, 1-\sum_{n=1}^{N-1}\left|v_{n}\right|^{2}>x\right\}, 0 \leq x \leq \frac{1}{N},
$$

which is a smaller simplex with volume (To see this point, note that the side length of this smaller simplex is $1-N x$.)

$$
(1-N x)^{N-1} \cdot \operatorname{Vol}(\mathcal{S})
$$

It follows from (75) and (77) that

$$
\mathbb{P}(u>x)=(1-N x)^{N-1} .
$$

Thus the pdf of $u$ is

$$
f_{u}(x)=\frac{-d \mathbb{P}(u>x)}{d x}=N(N-1)(1-N x)^{N-2}, \quad 0 \leq x \leq \frac{1}{N} .
$$

Comparing (74) and (79), we see that $\left|v_{n}\right|^{2}$ has the same distribution as $N u$ for all $n$.

Besides the MIMO system considered in the beginning of this section, which employs independent coding for each substream, we also consider a system where a single SISO Gaussian code is applied across $N$ substreams. With a linear MMSE equalizer, the D-M gain tradeoff of the latter system is

$$
\bar{d}_{\mathrm{mmse}}(r) \triangleq-\lim _{\mathrm{snr} \rightarrow \infty} \frac{\log \mathbb{P}\left(\sum_{n=1}^{N} \log \left(1+\rho_{\mathrm{mmse}, n}\right)<r \log \mathrm{snr}\right)}{\log \mathrm{snr}},
$$

while D-M tradeoff of the former system is $d_{\text {mmse }}(r)$ as defined in (67).

The following theorem establishes that the equality holds in (67).

Theorem V.2:

$$
\bar{d}_{\mathrm{mmse}}(r)=d_{\mathrm{mmse}}(r)=(M-N+1)\left(1-\frac{r}{N}\right)
$$


Proof: It is easy to see that (cf. (66))

$$
P_{\text {out }, \text { mmse }}(\text { snr }) \geq \mathbb{P}\left(\sum_{n=1}^{N} \log \left(1+\rho_{\text {mmse }, n}\right)<r \log \text { snr }\right) .
$$

Consequently,

$$
\bar{d}_{\mathrm{mmse}}(r) \geq d_{\mathrm{mmse}}(r) .
$$

Let us denote the sum of the mutual informations between channel input and the output over the $N$ substreams of an MMSE equalizer as

$$
I_{\text {mmse }}=\sum_{n=1}^{N} \log \left(1+\rho_{\text {mmse }, n}\right)=\sum_{n=1}^{N} \log \left(\frac{\mathrm{snr}}{\left[\left(\mathbf{H}^{*} \mathbf{H}+\frac{1}{\mathrm{snr}} \mathbf{I}\right)^{-1}\right]_{n n}}\right) .
$$

Let $\mathbf{H}^{*} \mathbf{H}=\mathbf{V} \boldsymbol{\Lambda}^{2} \mathbf{V}^{*}$ be its SVD with $\lambda_{1}^{2} \geq \cdots \geq \lambda_{N}^{2}$ as the ordered diagonal entries of $\boldsymbol{\Lambda}^{2}$. We can rewrite (84) as

$$
I_{\mathrm{mmse}}=\sum_{n=1}^{N} \log \left(\frac{\mathrm{snr}}{\mathbf{v}_{n}^{*}\left(\boldsymbol{\Lambda}^{2}+\frac{1}{\mathrm{snr}} \mathbf{I}\right)^{-1} \mathbf{v}_{n}}\right),
$$

where $\mathbf{v}_{n}$ is the $n$th column $\mathbf{V}^{*}$. Recall from Theorem II.2 that $\mathbf{V}$ is a Haar matrix and is independent of $\boldsymbol{\Lambda}$. Since

$$
\mathbf{v}_{n}^{*}\left(\boldsymbol{\Lambda}^{2}+\frac{1}{\mathrm{snr}} \mathbf{I}\right)^{-1} \mathbf{v}_{n}=\sum_{i=1}^{N}\left|v_{n i}\right|^{2}\left(\lambda_{i}^{2}+\mathrm{snr}^{-1}\right)^{-1} \geq\left|v_{n N}\right|^{2}\left(\lambda_{N}^{2}+\mathrm{snr}^{-1}\right)^{-1},
$$

where $v_{n i}$ is the $i$ th element of $\mathbf{v}_{n}^{*}$, the mutual information of the $n$th substream can be bounded as

$$
\log \left(\frac{\mathrm{snr}}{\mathbf{v}_{n}^{*}\left(\boldsymbol{\Lambda}^{2}+\frac{1}{\mathrm{snr}} \mathbf{I}\right)^{-1} \mathbf{v}_{n}}\right) \leq \log \left(\frac{1+\lambda_{N}^{2} \mathrm{snr}}{\left|v_{n N}\right|^{2}}\right), \quad n=1, \cdots, N,
$$

and hence

$$
I_{\mathrm{mmse}} \leq \sum_{n=1}^{N} \log \left(\frac{1+\lambda_{N}^{2} \mathrm{snr}}{\left|v_{n N}\right|^{2}}\right) .
$$

Hence, we can upper bound the D-M gain tradeoff

$$
\bar{d}_{\mathrm{mmse}}(r) \leq-\lim _{\mathrm{snr} \rightarrow \infty} \frac{\log \mathbb{P}\left(N \log \left(1+\lambda_{N}^{2} \mathrm{snr}\right)-\sum_{n=1}^{N} \log \left|v_{n N}\right|^{2}<r \log \mathrm{snr}\right)}{\log \mathrm{snr}} .
$$

Since $\left[v_{1 N} v_{2 N} \cdots v_{N N}\right]^{T}$ is a column of the Haar matrix $\mathbf{V}^{*},\left|v_{n N}\right|^{2}$ has the pdf given in (69), we can obtain after some routine calculations that

$$
\mathbb{E}\left[\log \left|v_{n N}\right|^{2}\right]=-\sum_{n=1}^{N-1} \frac{1}{n}
$$

Because $\left|v_{n N}\right|$ 's have the same pdf,

$$
\mathbb{E}\left[\sum_{n=1}^{N} \log \left|v_{n N}\right|^{2}\right]=-N \sum_{n=1}^{N-1} \frac{1}{n} \triangleq E,
$$


which is a negative constant. Next, consider the following set inequality which allows us to further lower bound $P_{\text {out,mmse }}(\mathrm{snr})$,

$$
\begin{aligned}
\left\{\lambda_{N},\left\{\left|v_{n N}\right|^{2}\right\}_{n=1}^{N}: N \log \left(1+\lambda_{N}^{2} \text { snr }\right)-\sum_{n=1}^{N} \log \left|v_{n N}\right|^{2}<r \log \mathrm{snr}\right\} \\
\supset\left\{\lambda_{N},\left\{\left|v_{n N}\right|^{2}\right\}_{n=1}^{N}: N \log \left(1+\lambda_{N}^{2} \mathrm{snr}\right)<r \log \mathrm{snr}+E\right\} \cap\left\{\sum_{n=1}^{N} \log \left|v_{n N}\right|^{2}>E\right\} .
\end{aligned}
$$

Using the statistical independence between $\lambda_{N}$ and $\left\{\left|v_{n N}\right|^{2}\right\}_{n=1}^{N}$ we have

$$
\begin{aligned}
& \mathbb{P}\left(N \log \left(1+\lambda_{N}^{2} \text { snr }\right)-\sum_{n=1}^{N} \log \left|v_{n N}\right|^{2}<r \log \text { snr }\right) \\
& \quad>\mathbb{P}\left(N \log \left(1+\lambda_{N}^{2} \text { snr }\right)<r \log \mathrm{snr}+E\right) \mathbb{P}\left(\sum_{n=1}^{N} \log \left|v_{n N}\right|^{2}>E\right) .
\end{aligned}
$$

Hence, (89) can be further bounded as

$$
\bar{d}_{\text {mmse }}(r) \leq-\lim _{\text {snr } \rightarrow \infty} \frac{\log \mathbb{P}\left(N \log \left(1+\lambda_{N}^{2} \text { snr }\right)<r \log \mathrm{snr}+E\right)+\log \mathbb{P}\left(\sum_{n=1}^{N} \log \left|v_{n N}\right|^{2}>E\right)}{\log \text { snr }} .
$$

Since $\mathbb{P}\left(\sum_{n=1}^{N} \log \left|v_{n N}\right|^{2}>E\right)$ is a positive constant independent of snr, we have

$$
\begin{aligned}
\bar{d}_{\mathrm{mmse}}(r) & \leq-\lim _{\mathrm{snr} \rightarrow \infty} \frac{\log \mathbb{P}\left(N \log \left(1+\lambda_{N}^{2} \mathrm{snr}\right)<r \log \mathrm{snr}+E\right)}{\log \mathrm{snr}} \\
& =-\lim _{\mathrm{snr} \rightarrow \infty} \frac{\log \mathbb{P}\left(N \log \left(1+\lambda_{N}^{2} \mathrm{snr}\right)<r \log \mathrm{snr}\right)}{\log \mathrm{snr}} \\
& =-\lim _{\mathrm{snr} \rightarrow \infty} \frac{\log \mathbb{P}\left(\lambda_{N}^{2}<\operatorname{snr}^{\frac{r}{N}-1}\right)}{\log \mathrm{snr}}
\end{aligned}
$$

By Theorem II.3, we obtain the upper bound

$$
\bar{d}_{\text {mmse }}(r) \leq(M-N+1)\left(1-\frac{r}{N}\right), \quad 1 \leq r \leq N
$$

Combining this with (67) and the bound (83), we have that the D-M tradeoff of MMSE is

$$
d_{\text {mmse }}(r)=\bar{d}_{\text {mmse }}(r)=(M-N+1)\left(1-\frac{r}{N}\right)
$$

and the theorem is proved.

At first glance, the conclusion that even considering the lower bound on outage probability in (82) does not improve system diversity gain is rather surprising. Since the $N$ substreams usually have distinct output SNRs (even if they have identical marginal distributions), it seems unlikely that all the $N$ substreams are in outage simultaneously. Note that $I_{\text {mmse }}$ is the mutual information rate realized 
in a parallel channel that results from fixing the receiver front-end to be the linear MMSE equalizer with the transmitter using this knowledge to code across the $N$ substreams. One might expect to achieve an $N$-fold diversity gain with such coding compared to using independent coding across the antennas. Theorem V.2 however implies that the output SNRs of the $N$ substreams are actually highly correlated. This point can be seen from (87); if $\lambda_{N} \leq \mathrm{snr}^{-1}$, then the mutual informations in all the $N$ substreams tend to be small.

Corollary V.3: For both ZF and MMSE, the $N$ substreams, ranking from the strongest to the weakest, have diversity gain of their individual outage probabilities to be all equal to $M-N+1$.

Proof: We first consider the MMSE case. Suppose that the strongest substream, with output SNR $\rho_{\max }$, has diversity gain $d_{\max }>M-N+1$. According to $(96), \bar{d}_{\text {mmse }}(0)=M-N+1$. Denote the mutual information $I_{\text {mmse }}(\mathrm{snr}) \triangleq \sum_{n=1}^{N} \log \left(1+\rho_{\text {mmse }, n}\right) \geq \log \left(1+\rho_{\max }\right)$. Then

$$
-\lim _{\text {snr } \rightarrow \infty} \frac{\log \mathbb{P}\left(I_{\text {mmse }}(\text { snr })<\text { const }\right)}{\log \text { snr }} \geq-\lim _{\text {snr } \rightarrow \infty} \frac{\log \mathbb{P}\left(\log \left(1+\rho_{\max }\right)<\text { const }\right)}{\log \text { snr }}=d_{\max }>M-N+1,
$$

where const stands for a finite constant. Note that the leftmost term of the above equation is equal to $\bar{d}_{\text {mmse }}(0)=M-N+1$, which leads to a contradiction. Thus $d_{\max } \leq M-N+1$. On the other hand, suppose the weakest substream has diversity gain $d_{\min }<M-N+1$. With the same but independent coding applied to all the substreams, the overall system outage probability would be dominated by that of the weakest substream, which is of order $\mathrm{snr}^{-d_{\min }}$. It implies that $d_{\mathrm{mmse}}(0)<M-N+1$, which also leads to a contradiction. Therefore $d_{\min } \geq M-N+1$, and we conclude that all the substreams must have the same diversity gain $M-N+1$ for the linear MMSE equalizer. The argument for the case of ZF is straightforward given the above.

\section{D-M Gain Tradeoff of V-BLAST with Channel-Dependent Ordered Detection}

Based on Corollary V.3, we are ready to answer the long standing open question as to what really is the D-M tradeoff of V-BLAST with channel-dependent ordered decoding.

In contrast to the linear equalizers which decode the $N$ substreams simultaneously, the V-BLAST equalizer applies successive nulling and interference cancellation to recover the substreams one by one. At each step, the V-BLAST estimates one substream according to the criteria of ZF or MMSE and then eliminates the estimated component from the received data. Hence at the next step, the substream to detect is subject to one less interferer [29]. We refer to the V-BLAST based on the ZF and MMSE criteria as the ZF-VB and MMSE-VB, respectively. The output SNRs of the substreams estimated by 
the ZF-VB or MMSE-VB are closely related to applying the QR decomposition to the channel matrix. In particular, denoting the QR decomposition $\mathbf{H}=\mathbf{Q R}$, the ZF-VB yields $N$ substreams with output SNRs (cf. [3], [13])

$$
\rho_{\mathrm{zf}-\mathrm{vb}, n}=r_{n n}^{2} \mathrm{snr} \quad \text { for } \quad 1 \leq n \leq N
$$

where $r_{n n}, n=1, \ldots, N$ are the diagonal entries of $\mathbf{R} .{ }^{6}$ Similarly, the MMSE-VB yields $N$ substreams with [29], [30]

$$
\rho_{\mathrm{mmse}-\mathrm{vb}, n}=\breve{r}_{n n}^{2} \mathrm{snr}-1 \text { for } \quad 1 \leq n \leq N,
$$

where $\breve{r}_{n n}, n=1, \ldots, N$ are the diagonal entries of $\breve{\mathbf{R}}$ yielded by the QR decomposition $\left(\begin{array}{c}\mathbf{H} \\ \text { snr }^{-\frac{1}{2}} \mathbf{I}\end{array}\right)=$

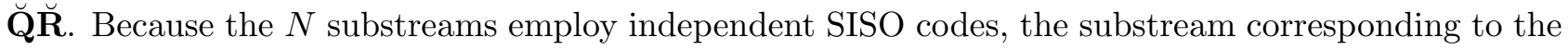
lowest output SNR is the bottleneck of the overall system [31]. One remedy of this undesirable effect is to apply channel-dependent ordering [32][2]. The channel dependent ordering can be represented by a permutation matrix $\boldsymbol{\Pi}$ (this is actually a function of $\mathbf{H}$ but we don't write $\boldsymbol{\Pi}(\mathbf{H})$, for simplicity) and the output SNRs of the substreams obtained via ordered V-BLAST are therefore dependent on the QR decompositions $\mathbf{H \Pi}=\mathbf{Q R}$ and $\left(\begin{array}{c}\mathbf{H \Pi} \\ \text { snr }^{-\frac{1}{2}} \mathbf{I}\end{array}\right)=\breve{\mathbf{Q}} \breve{\mathbf{R}}$. The permutation matrix is chosen such that $\min _{1 \leq n \leq N}\left\{r_{n n}^{2}\right\}$ (or $\min _{1 \leq n \leq N}\left\{\breve{r}_{n n}^{2}\right\}$ in the MMSE-VB case) is maximized among the $N$ ! permutations. Define $r_{\max }^{2} \triangleq \max _{\Pi} \min _{1 \leq n \leq N}\left\{r_{n n}^{2}\right\}$ and $r_{\min }^{2} \triangleq \min _{\Pi} \min _{1 \leq n \leq N}\left\{r_{n n}^{2}\right\}$. And $\breve{r}_{\max }^{2}$ and $\breve{r}_{\min }^{2}$ are similarly defined with $r_{n n}^{2}$ replaced by $\breve{r}_{n n}^{2}$.

Then with any ordered detection the D-M tradeoffs of ZF-VB and MMSE-VB are sandwiched by

$$
-\lim _{\mathrm{snr} \rightarrow \infty} \frac{\log \mathbb{P}\left(\log \left(1+r_{\min }^{2} \mathrm{snr}\right)<\frac{r}{N} \log \mathrm{snr}\right)}{\log \mathrm{snr}} \leq d_{\mathrm{zf}-\mathrm{vb}}(r) \leq-\lim _{\mathrm{snr} \rightarrow \infty} \frac{\log \mathbb{P}\left(\log \left(1+r_{\max }^{2} \mathrm{snr}\right)<\frac{r}{N} \log \mathrm{snr}\right)}{\log \mathrm{snr}}
$$

and

$$
-\lim _{\mathrm{snr} \rightarrow \infty} \frac{\log \mathbb{P}\left(\log \left(\breve{r}_{\min }^{2} \mathrm{snr}\right)<\frac{r}{N} \log \mathrm{snr}\right)}{\log \mathrm{snr}} \leq d_{\mathrm{mmse}-\mathrm{vb}}(r) \leq-\lim _{\mathrm{snr} \rightarrow \infty} \frac{\log \mathbb{P}\left(\log \left(\breve{r}_{\max }^{2} \mathrm{snr}\right)<\frac{r}{N} \log \mathrm{snr}\right)}{\log \mathrm{snr}},
$$

respectively. It is not difficult to show that with detection ordering $\boldsymbol{\Pi}$

$$
r_{N N}^{2}=\frac{1}{\left[\left(\boldsymbol{\Pi}^{T} \mathbf{H}^{*} \mathbf{H} \Pi\right)^{-1}\right]_{N N}} \quad \text { and } \quad \breve{r}_{N N}^{2}=\frac{1}{\left[\left(\boldsymbol{\Pi}^{T} \mathbf{H}^{*} \mathbf{H} \boldsymbol{\Pi}+\frac{1}{\mathrm{snr}} \mathbf{I}\right)^{-1}\right]_{N N}} .
$$

\footnotetext{
${ }^{6}$ To make the QR decomposition unique, the diagonal entries of $\mathbf{R}$ are positive.
} 
By choosing different $\Pi, r_{N N}^{2}$ and $\breve{r}_{N N}^{2}$ can take on one of $N$ different values:

$$
r_{N N}^{2}=\frac{1}{\left[\left(\mathbf{H}^{*} \mathbf{H}\right)^{-1}\right]_{n n}} \quad \text { and } \quad \breve{r}_{N N}^{2}=\frac{1}{\left[\left(\mathbf{H}^{*} \mathbf{H}+\frac{1}{\mathrm{snr}} \mathbf{I}\right)^{-1}\right]_{n n}}, 1 \leq n \leq N
$$

Comparing (97) (98) to (12) (13) and invoking (102), we see that the first detected substream using ZF-VB (or MMSE-VB) has the output SNR taken from $\rho_{\mathrm{zf}, n}$ (or $\rho_{\mathrm{mmse}, n}$ ), for $1 \leq n \leq N$. Define $r_{N N, \text { max }}^{2} \triangleq \max _{1 \leq n \leq N} \frac{1}{\left[\left(\mathbf{H}^{*} \mathbf{H}\right)^{-1}\right]_{n n}}$ and $r_{N N \text {,min }}^{2} \triangleq \min _{1 \leq n \leq N} \frac{1}{\left[\left(\mathbf{H}^{*} \mathbf{H}\right)^{-1}\right]_{n n}}$, while $\breve{r}_{N N, \text { max }}^{2}$ and $\breve{r}_{N N \text {,min }}^{2}$ are defined similarly. We show next that

$$
r_{\max }^{2} \leq r_{N N, \max }^{2} \text { and } r_{\min }^{2}=r_{N N, \min }^{2}
$$

By definition,

$$
r_{\max }^{2}=\max _{\Pi} \min _{1 \leq n \leq N}\left\{r_{n n}^{2}\right\} \leq \max _{\Pi}\left\{r_{N N}^{2}\right\}=\max _{1 \leq n \leq N} \frac{1}{\left[\left(\mathbf{H}^{*} \mathbf{H}\right)^{-1}\right]_{n n}}=r_{N N, \max }^{2}
$$

Hence the first inequality of (103) is proven. Again by definition, we can prove that

$$
r_{\min }^{2}=\min _{\Pi} \min _{1 \leq n \leq N}\left\{r_{n n}^{2}\right\} \leq \min _{\Pi}\left\{r_{N N}^{2}\right\}=r_{N N, \min }^{2}
$$

Moreover, suppose $r_{\min }^{2}<r_{N N \text {,min }}^{2}$, which means that for some permutation $\Pi$, $r_{\min }^{2}=r_{n n}^{2}$ for some $n \neq N$. Then we can always find a new permutation $\tilde{\boldsymbol{\Pi}}$ matrix such that the $n$th column of $\mathbf{H \Pi}$ is moved to the $N$ th column of $\mathbf{H} \tilde{\Pi}$, and the QR decomposition $\mathbf{H} \tilde{\boldsymbol{\Pi}}=\mathbf{Q} \tilde{\mathbf{R}}$ yields $\tilde{r}_{N N}^{2} \leq r_{n n}^{2}=r_{\min }^{2}<$ $r_{N N \text {, min }}^{2}$, where $\tilde{r}_{N N}^{2} \leq r_{n n}^{2}$ because moving the column to the right always reduces its corresponding $r_{i i}$ as it has more interference to suppress. Now we have reached a contradiction and hence proven that $r_{\min }^{2}=r_{N N \text {,min }}^{2}$. Using the same argument, we can prove that

$$
\breve{r}_{\max }^{2} \leq \breve{r}_{N N, \max }^{2} \text { and } \breve{r}_{\text {min }}^{2}=\breve{r}_{N N, \min }^{2}
$$

Let us first focus on the MMSE-VB case. Recall that the V-BLAST applies the same but independent coding. Therefore, with spatial multiplexing gain $r$, the outage probability of V-BLAST is [31]

$$
\mathbb{P}\left(\log \left(\min _{1 \leq n \leq N} \breve{r}_{n n}^{2} \mathrm{snr}\right)<\frac{r}{N} \log \mathrm{snr}\right)
$$

According to (100) and (106), we obtain that

$$
-\lim _{\mathrm{snr} \rightarrow \infty} \frac{\log \mathbb{P}\left(\log \left(\breve{r}_{N N, \min }^{2} \mathrm{snr}\right)<\frac{r}{N} \log \mathrm{snr}\right)}{\log \mathrm{snr}} \leq d_{\mathrm{mmse}-\mathrm{vb}}(r) \leq-\lim _{\mathrm{snr} \rightarrow \infty} \frac{\log \mathbb{P}\left(\log \left(\breve{r}_{N N, \mathrm{max}}^{2} \mathrm{snr}\right)<\frac{r}{N} \log \mathrm{snr}\right)}{\log \mathrm{snr}} .
$$


or equivalently,

$$
-\lim _{\mathrm{snr} \rightarrow \infty} \frac{\log \mathbb{P}\left(\breve{r}_{N N, \min }^{2} \leq \operatorname{snr}^{\frac{r}{N}-1}\right)}{\log \mathrm{snr}} \leq d_{\mathrm{mmse}-\mathrm{vb}}(r) \leq-\lim _{\mathrm{snr} \rightarrow \infty} \frac{\log \mathbb{P}\left(\breve{r}_{N N, \max }^{2} \leq \operatorname{snr}^{\frac{r}{N}-1}\right)}{\log \mathrm{snr}} .
$$

By Corollary V.3, we have

$$
-\lim _{\mathrm{snr} \rightarrow \infty} \frac{\log \mathbb{P}\left(\breve{r}_{N N, \min }^{2} \leq \operatorname{snr} \frac{r}{N}-1\right)}{\log \mathrm{snr}}=-\lim _{\mathrm{snr} \rightarrow \infty} \frac{\log \mathbb{P}\left(\breve{r}_{N N, \max }^{2} \leq \mathrm{snr} \frac{r}{N}-1\right)}{\log \mathrm{snr}}=(M-N+1)\left(1-\frac{r}{N}\right) .
$$

Hence, for any channel-dependent detection ordering $\boldsymbol{\Pi}$

$$
d_{\text {mmse-vb }}(r)=(M-N+1)\left(1-\frac{r}{N}\right), \quad 0 \leq r \leq N .
$$

Clearly, in similar vein, we can obtain

$$
d_{\mathrm{zf}-\mathrm{vb}}(r)=(M-N+1)\left(1-\frac{r}{N}\right), \quad 0 \leq r \leq N
$$

Now we have established the following theorem.

Theorem V.4: For both ZF-VB and MMSE-VB with any channel-dependent detection ordering, the D-M gain tradeoff of the overall system is

$$
d_{\mathrm{vb}}(r) \triangleq d_{\mathrm{mmse}-\mathrm{vb}}(r)=d_{\mathrm{zf}-\mathrm{vb}}(r)=(M-N+1)\left(1-\frac{r}{N}\right), \quad 0 \leq r \leq N .
$$

Theorem V.4 stands for the final answer to the long-standing open problem on whether optimal ordering in V-BLAST improves system diversity gain. Our answer is "no".

The result that the maximal diversity gain of the ZF-VB is $M-N+1$ even with detection ordering was first established in the conference version of this paper [11]. This result was also reached by Zhang et. al. in [33], where they further "predict that the whole diversity multiplexing tradeoff curve will not be improved by optimal ordering". However, their result can not be extended to the MMSE-VB case.

We conclude this section with the following corollary.

Corollary V.5: In the asymptotically high SNR regime, the overall outage probability of V-BLAST is dominated by that of the first detected layer for any detection ordering, .

Proof: Suppose there exists an ordering technique $\mathcal{T}$ which yields the $n$-th $(n \geq 2)$ detected layer with diversity gain $\mathrm{D} \leq M-N+1$, i.e., its outage probability $P_{\text {out }}(\mathrm{snr}) \propto \mathrm{snr}^{-\mathrm{D}}$ in the high SNR regime. With a random detection ordering, there is $\frac{1}{N !}$ chance that the random ordering coincides with $\mathcal{T}$. Hence the outage probability of the $n$-th layer is no less than $\frac{1}{N !} P_{\text {out }}($ snr $)$ and therefore its 
diversity gain is no greater than $\mathrm{D} \leq M-N+1$. However it is well known that the V-BLAST with random detection ordering yields the $n$-th detected layer with diversity gain $M-N+n$ [14], which is strictly greater than $\mathrm{D}$, which leads to a contradiction. Hence for any ordering technique, the $n$-th $(n \geq 2)$ detected layer has diversity gain strictly greater than $M-N+1$. Since the first detected layer has diversity gain $M-N+1$ by Theorem V.4, the corollary has been proven

\section{Vi. A Closer Look: Outage Probability and Coding Gain}

In this section, we consider the case where independent coding is applied to the $N$ substreams. We analyze the outage probability of the substreams. Despite the pessimistic conclusion of Section V with respect to the diversity gain, we show that there is a remarkable SNR gain due to applying the optimal detection ordering in the V-BLAST architecture which we quantify next.

To facilitate the analysis, we rewrite the output SNRs of ZF

$$
\rho_{\mathrm{zf}, n}=\frac{\mathrm{snr}}{\left[\left(\mathbf{H}^{*} \mathbf{H}\right)^{-1}\right]_{n n}},
$$

and MMSE

$$
\rho_{\text {mmse }, n}=\frac{\text { snr }}{\left[\left(\mathbf{H}^{*} \mathbf{H}+\mathbf{s n r} \mathbf{I}\right)^{-1}\right]_{n n}}-1 .
$$

According to (86) we have the upper bound

$$
\rho_{\text {mmse }, n} \leq \frac{\mathrm{snr}}{\left|v_{n N}\right|^{2}\left(\lambda_{N}^{2}+\mathrm{snr}^{-1}\right)^{-1}}-1 .
$$

Similarly, we obtain

$$
\rho_{\mathrm{zf}, n} \leq \frac{\lambda_{N}^{2} \mathrm{snr}}{\left|v_{n N}\right|^{2}} .
$$

Given the target rate $R$ of the $n$th substream, its outage probability is

$$
\begin{aligned}
P_{\text {out }, n}^{\mathrm{zf}}(R, \mathrm{snr}) & =\mathbb{P}\left(\log \left(1+\rho_{\mathrm{zf}, n}\right)<R\right) \\
& =\mathbb{P}\left(\frac{\mathrm{snr}}{\sum_{i=1}^{N}\left|v_{n i}\right|^{2} \lambda_{i}^{-2}}<2^{R}-1\right) \\
& \geq \mathbb{P}\left(\frac{\mathrm{snr}}{\left|v_{n N}\right|^{2} \lambda_{N}^{-2}}<2^{R}-1\right) \triangleq \underline{P_{\text {out }, n}^{\mathrm{zf}}}(R, \text { snr }) .
\end{aligned}
$$

if $\mathrm{ZF}$ is used, and for MMSE

$$
\begin{aligned}
P_{\text {out }, n}^{\mathrm{mmse}}(R, \mathrm{snr}) & =\mathbb{P}\left(\log \left(1+\rho_{\mathrm{mmse}, n}\right)<R\right) \\
& =\mathbb{P}\left(\frac{\mathrm{snr}}{\sum_{i=1}^{N}\left|v_{n i}\right|^{2}\left(\lambda_{i}^{2}+\mathrm{snr}^{-1}\right)^{-1}}<2^{R}\right) \\
& \geq \mathbb{P}\left(\frac{\mathrm{snr}}{\left|v_{n N}\right|^{2}\left(\lambda_{N}^{2}+\mathrm{snr}^{-1}\right)^{-1}}<2^{R}\right) \triangleq \underline{P_{\text {out }, n}^{\mathrm{mmse}}}(R, \mathrm{snr}),
\end{aligned}
$$


With regard to the lower bounds (118) and (120), we have the following lemma.

Lemma VI.1:

$$
\lim _{\mathrm{snr} \rightarrow \infty} \frac{P_{\mathrm{out}, n}^{\mathrm{zf}}(R, \mathrm{snr})}{P_{\mathrm{out}, n}^{\mathrm{zf}}(R, \mathrm{snr})}=1,
$$

and

$$
\lim _{\text {snr } \rightarrow \infty} \frac{P_{\text {out }, n}^{\mathrm{mmse}}(R, \mathrm{snr})}{P_{\mathrm{out}, n}^{\mathrm{mmse}}(R, \mathrm{snr})}=1 .
$$

That is, the lower bounds in (118) and (120) are asymptotically tight at high SNR.

Proof: The proof is rather technical. We relegate it to Appendix B.

We are now ready to establish the following theorem which quantifies the SNR gain that accrues by applying the optimal decoding ordering to the V-BLAST architecture.

Theorem VI.2: Given the input SNR snr and the target rate $R$ for each substream. Denote $P_{\text {out,min }}^{\mathrm{zf}}(R, \mathrm{snr})$ and $P_{\text {out,min }}^{\text {mmse }}(R$, snr $)$ the outage probabilities of the substreams with the highest output SNR obtained using ZF and MMSE, respectively. Then in the high SNR regime,

$$
P_{\text {out }, \min }^{\mathrm{zf}}\left(R, \frac{\mathrm{snr}}{N}\right) \simeq P_{\text {out }, n}^{\mathrm{zf}}(R, \mathrm{snr}), \quad \forall n,
$$

and

$$
P_{\text {out }, \text { min }}^{\mathrm{mmse}}\left(R, \frac{\mathrm{snr}}{N}\right)<P_{\text {out }, n}^{\mathrm{mmse}}(R, \mathrm{snr}), \quad \forall n .
$$

where the approximation in (123) is asymptotically accurate as $\mathrm{snr} \rightarrow \infty$ (in the sense that the limit of the ratio of the two probabilities tends to unity with increasing SNR). Comparing to the fixed decoding order, applying the optimal decoding order yields $10 \log _{10} N \mathrm{~dB}$ SNR gain for ZF-VB, and more than $10 \log _{10} N$ dB SNR gain for MMSE-VB.

Proof: Due to (121) and (122), we can closely approximate the outage probability of the $n$th substream by

$$
P_{\mathrm{out}, n}^{\mathrm{zf}}(R, \mathrm{snr}) \simeq \mathbb{P}\left(\frac{\lambda_{N}^{2}}{\left|v_{n N}\right|^{2}}<\frac{2^{R}-1}{\mathrm{snr}}\right) \triangleq \underline{P_{\mathrm{out}, n}^{\mathrm{zf}}}(R, \mathrm{snr}),
$$

and

$$
P_{\mathrm{out}, n}^{\mathrm{mmse}}(R, \mathrm{snr}) \simeq \mathbb{P}\left(\frac{\lambda_{N}^{2}+\mathrm{snr}^{-1}}{\left|v_{n N}\right|^{2}}<\frac{2^{R}}{\mathrm{snr}}\right) \triangleq \underline{P_{\mathrm{out}, n}^{\mathrm{mmse}}}(R, \mathrm{snr}),
$$

at high SNR. Define $u \triangleq \min \left\{\left|v_{n N}\right|^{2}, 1 \leq n \leq N\right\}$. Then

$$
P_{\text {out }, \text { min }}^{\mathrm{zf}}(R, \mathrm{snr}) \simeq \mathbb{P}\left(\frac{\lambda_{N}^{2}}{u}<\frac{2^{R}-1}{\mathrm{snr}}\right) \triangleq \underline{P_{\mathrm{out}, \min }^{\mathrm{zf}}}(R, \mathrm{snr}),
$$

and

$$
P_{\text {out,min }}^{\text {mmse }}(R, \text { snr }) \simeq \mathbb{P}\left(\frac{\lambda_{N}^{2}+\mathrm{snr}^{-1}}{u}<\frac{2^{R}}{\mathrm{snr}}\right) \triangleq \underline{P_{\text {out,min }}^{\text {mmse }}}(R, \text { snr }) .
$$


Recall from Lemma V.1 that $\left|v_{n N}\right|^{2} \sim N u, \forall n$, which implies that

$$
\mathbb{P}\left(\frac{\lambda_{N}^{2}}{u}<\frac{2^{R}-1}{\mathrm{snr} / N}\right)=\mathbb{P}\left(\frac{\lambda_{N}^{2}}{\left|v_{n N}\right|^{2}}<\frac{2^{R}-1}{\mathrm{snr}}\right) .
$$

It follows from (129), (127), and (125) that

$$
\underline{P_{\text {out }, \text { min }}^{\mathrm{zf}}}\left(R, \frac{\mathrm{snr}}{N}\right)=\underline{P_{\mathrm{out}, n}^{\mathrm{zf}}}(R, \mathrm{snr}),
$$

and hence

$$
P_{\text {out }, \min }^{\mathrm{zf}}\left(R, \frac{\mathrm{snr}}{N}\right) \simeq P_{\text {out }, n}^{\mathrm{zf}}(R, \mathrm{snr}) .
$$

Because the approximations in (127) and (125) are asymptotically accurate as snr $\rightarrow \infty$, so is the approximation in (131). Therefore with respect to ZF, we can conclude that the strongest substream has $10 \log _{10} N \mathrm{~dB}$ SNR gain over an average one. Because V-BLAST applies independent coding to each layer, V-BLAST is in outage if and only if at least one layer is in outage. It is known from Corollary V.5 that in the high SNR regime, the outage events of V-BLAST are dominated by those of the first detected layer. Consequently, the ZF-VB with the optimal detection ordering has $10 \log _{10} N$ dB SNR gain over ZF-VB with fixed detection ordering.

As for MMSE, according to (128),

$$
\begin{aligned}
\underline{P_{\text {out }, \text { min }}^{\text {mmse }}\left(R, \frac{\mathrm{snr}}{N}\right)} & =\mathbb{P}\left(1+\lambda_{N}^{2} \frac{\mathrm{snr}}{N}<2^{R} \cdot u\right) \\
& =\mathbb{P}\left(N+\lambda_{N}^{2} \mathrm{snr}<2^{R}\left|v_{N n}\right|^{2}\right) \\
& <\mathbb{P}\left(1+\lambda_{N}^{2} \mathrm{snr}<2^{R}\left|v_{N n}\right|^{2}\right)=\underline{P_{\text {out }, n}^{\mathrm{mmse}}}(R, \mathrm{snr}) .
\end{aligned}
$$

As the approximations in (126) and (128) are asymptotically accurate at high SNR, we can see that

$$
P_{\text {out }, \text { min }}^{\text {mmse }}\left(R, \frac{\mathrm{snr}}{N}\right)<P_{\text {out }, n}^{\text {mmse }}(R, \text { snr }),
$$

which implies that the SNR gain of strongest substream over an average substream is more than $10 \log _{10} N$ dB. Moreover, it can be seen that the gap between (133) and (134) gets larger as $N$ increases.

Finally, we remark that the key fact used to determine the coding gain advantage of ordered detection is that $\left|v_{n N}\right|^{2} \sim N u$, where $v_{n N}$ is the $(n, N)$ th entry of the unitary matrix $\mathbf{V}^{*}$, and $u=\min \left\{\left|v_{n N}\right|^{2}, n=1, \cdots, N\right\}$. According to Lemma V.1, $\left|v_{n N}\right|^{2} \sim N u$ as long as $\mathbf{V}$ is a Haar matrix. If the columns of $\mathbf{H}$ are independent, then the distribution of $\mathbf{H}$ is invariant under the right multiplication of a unitary matrix. Hence $\mathbf{V}$ is a Haar matrix. We see that Theorem VI.2 still holds even the rows of $\mathbf{H}$ are correlated but the columns of $\mathbf{H}$ are statistically independent. 


\section{Numerical EXAmples}

In this section, we present several numerical examples to validate the preceding theoretical analysis.

Figure 1 presents the quantile-quantile (qq) figures for the distribution of $\frac{M-N+2}{N-1} \eta_{\text {snr }}$ against $\frac{M-N+2}{N-1} \eta_{\infty}$ which is of $\mathcal{F}$-distribution (see 35 ). We see that the $F$-distribution approximation works very well for all cases in the high SNR regime. We also see that the approximation is less accurate when $N=M$. The explanation is as follows. It is known that for $M=N$, the channel matrix tends to be more ill-conditioned thus the smallest diagonal entry of $\boldsymbol{\Lambda}_{n}$ (see (26)) is close to zero. Therefore the convergence of (26) to the limit (28) is slower. For the case of $N=M / 2$, the $F$-distribution approximation is very accurate even for a moderate SNR of $10 \mathrm{~dB}$.
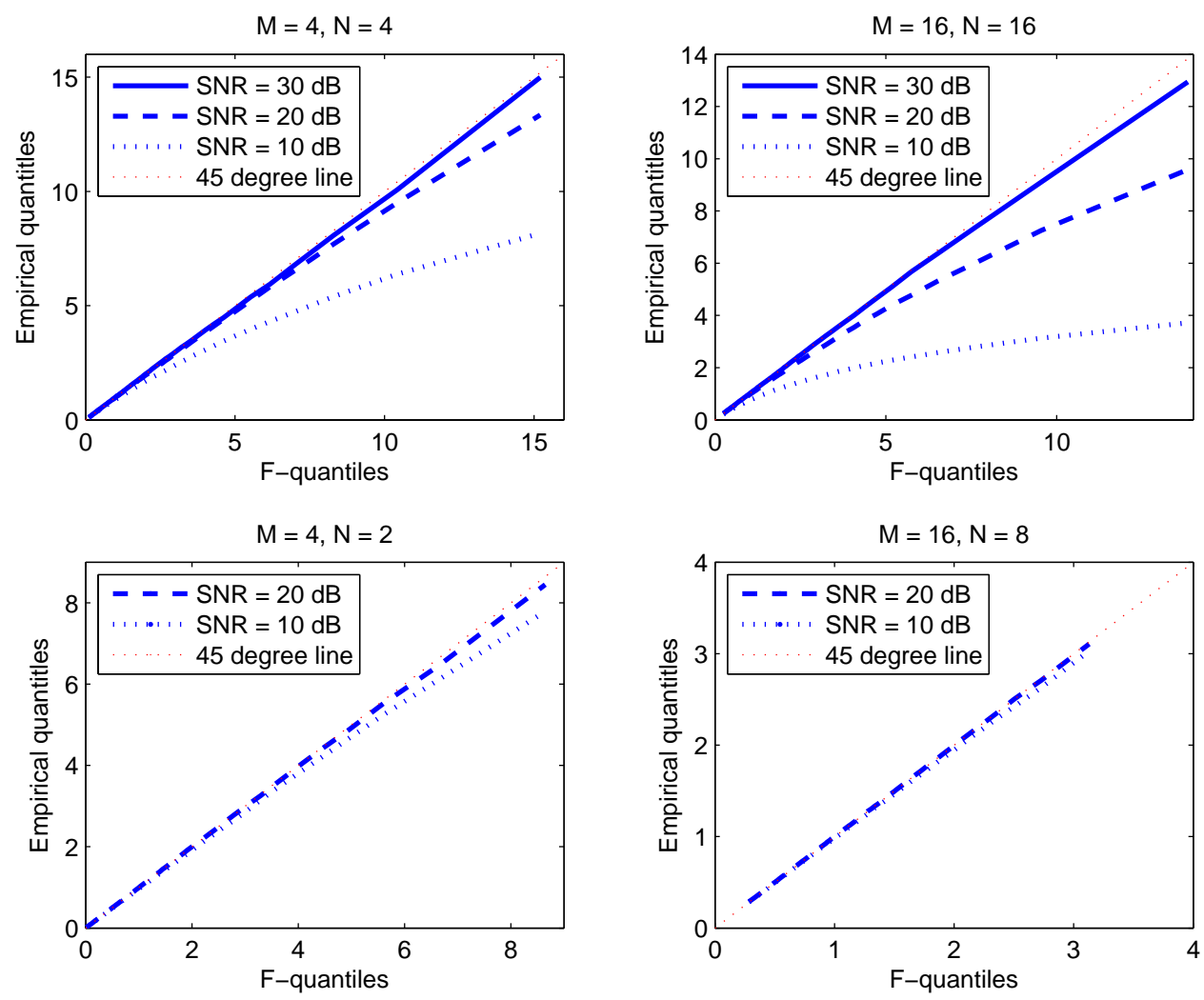

Fig. 1. Quantile-quantile plots for $\frac{M-N+2}{N-1} \eta_{\text {snr. }}$. The range of the quantiles is $1 \%-99 \%$.

In the second example, we consider an i.i.d. Rayleigh channel with $M=5$ and $N=4$. We calculate the INRs in the output of MMSE based on $10^{4}$ Monte Carlo trials. Figure 2 shows the INRs at different input SNRs, in which each curve represents the $10^{4}$ INRs sorted in the non-decreasing order. This simulation result agrees with Lemma III.2; the INR is inversely proportional to the input SNR.

Figure 3 compares the uncoded error probabilities of ZF and MMSE equalizers in a $4 \times 4$ channel with 


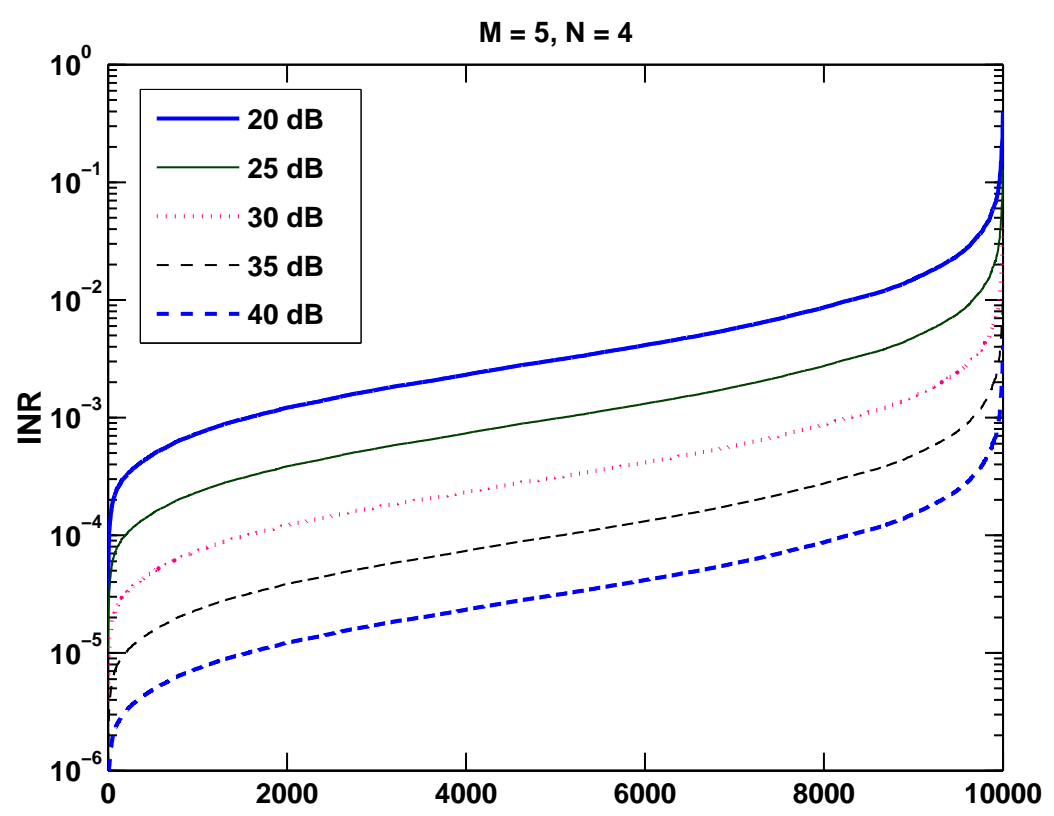

Fig. 2. INRs in the output of MMSE with input SNR equal to 20, 25, 30, 35, and $40 \mathrm{~dB}$. The results are based on $10^{4}$ Monte Carlo trials of the channel matrix. $M=5, N=4$.

BPSK input. We see that the error probabilities of MMSE obtained via averaging over $10^{5}$ Monte Carlo simulations match extremely well with the high SNR approximation of (46) for a moderate SNR (snr $\geq 10 \mathrm{~dB}$ ). Moreover, as predicted in (50), there is a non-vanishing gap between the error probability curves of MMSE and ZF.

Figure 4 compares the outage probabilities of ZF and MMSE equalizers in an i.i.d. Rayleigh channel with $M=6$ and $N=5$. We consider the three cases where the target rates are one, two, and four bps $/ \mathrm{Hz}$ per substream. Similar to Figure 3, the outage probabilities of MMSE obtained via averaging over $10^{7}$ Monte Carlo simulations (represented by +") match exactly at high SNR with the approximation in (52) which is obtained via numerical integration (represented in solid lines). Although for fixed $R$ MMSE has a non-vanishing SNR gain over ZF, the gap becomes smaller as $R$ increases. Figure 4 also illustrates that the $\epsilon$-outage capacity is the same for MMSE and ZF at asymptotically high SNR.

In the fifth example, we consider a channel with $M=N=3$. Suppose $N$ independent capacityachieving codes are applied to each substream, and the target rate is $R=3 \mathrm{bps} / \mathrm{Hz}$ for each substream. The four solid lines in Figure 5, from top to bottom, represent $P_{\mathrm{out}, n}^{\mathrm{zf}}, P_{\mathrm{out}, n}^{\mathrm{mmse}}, P_{\mathrm{out}, \mathrm{zf} i n}^{\mathrm{zf}}$, and $P_{\mathrm{out}, \mathrm{min}}^{\mathrm{mm}}$, respectively. The four dashed lines underneath the solid lines are the corresponding lower bounds, i.e., $\underline{P_{\text {out }, n}^{\mathrm{zf}}}, \underline{P_{\mathrm{out}, n}^{\mathrm{mm} e}}, \underline{P_{\mathrm{out}, \mathrm{min}}^{\mathrm{zf}}}$, and $\underline{P_{\mathrm{out}, \mathrm{min}}^{\mathrm{mmse}}}$. We see from Figure 5 that (i) all the lower bounds are 


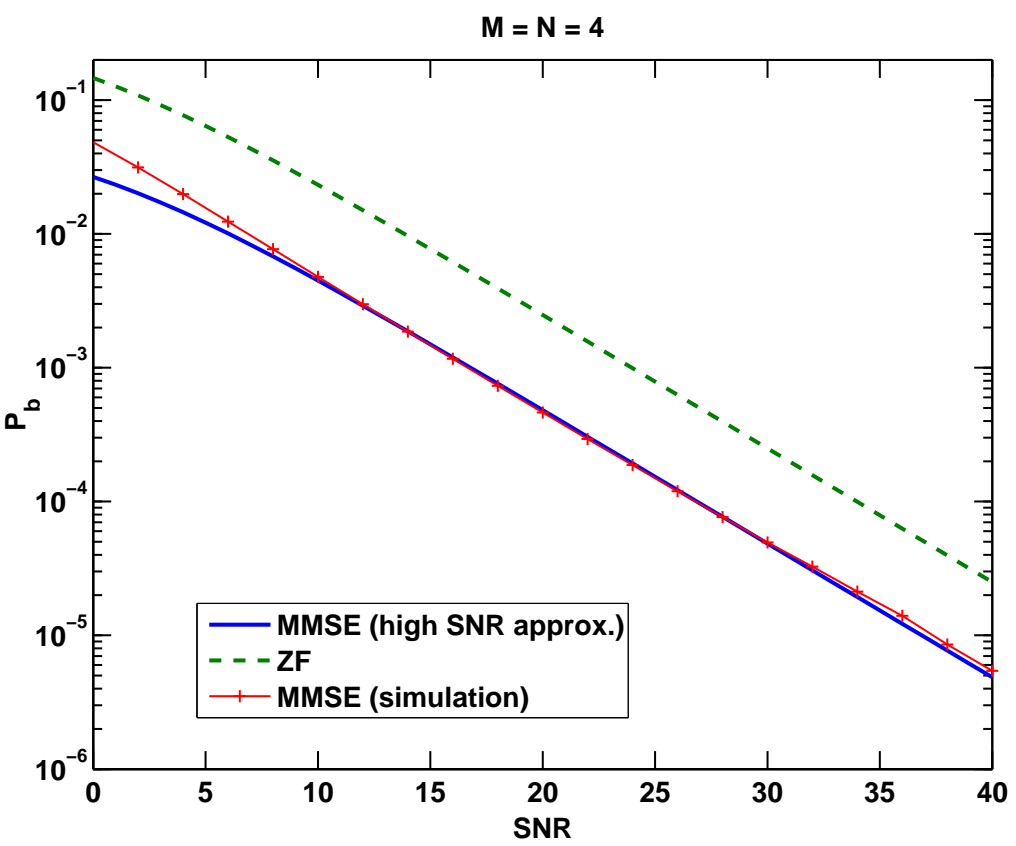

Fig. 3. Error probabilities of ZF given in (39) and MMSE (Monte Carlo trials and high SNR approximation given in (46)). $M=N=4$.

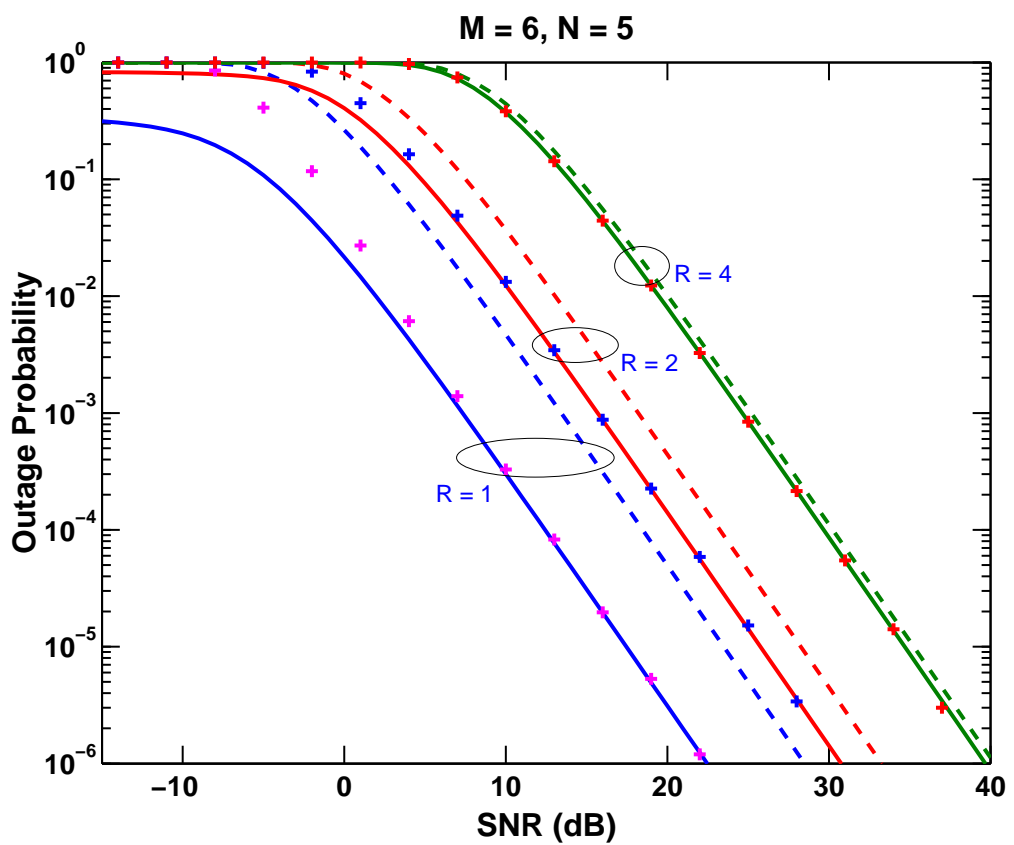

Fig. 4. Outage probabilities of ZF given in (51) (represented by dashed lines) and MMSE via Monte Carlo trials $(+)$ and high SNR approximation given in (52) (solid line). $M=6, N=5$.

asymptotically tight as SNR increases, (ii) the gap between $P_{\text {out,min }}^{\mathrm{zf}}$ and $P_{\text {out, } n}^{\mathrm{zf}}$ is $10 \log _{10} N=4.77$ $\mathrm{dB}$, (iii) the coding gain of $P_{\mathrm{out}, \text { min }}^{\text {mmse }}$ over $P_{\mathrm{out}, n}^{\mathrm{mmse}}$ is significantly larger than $4.77 \mathrm{~dB}$. This numerical 
example agrees with Theorem VI.2.

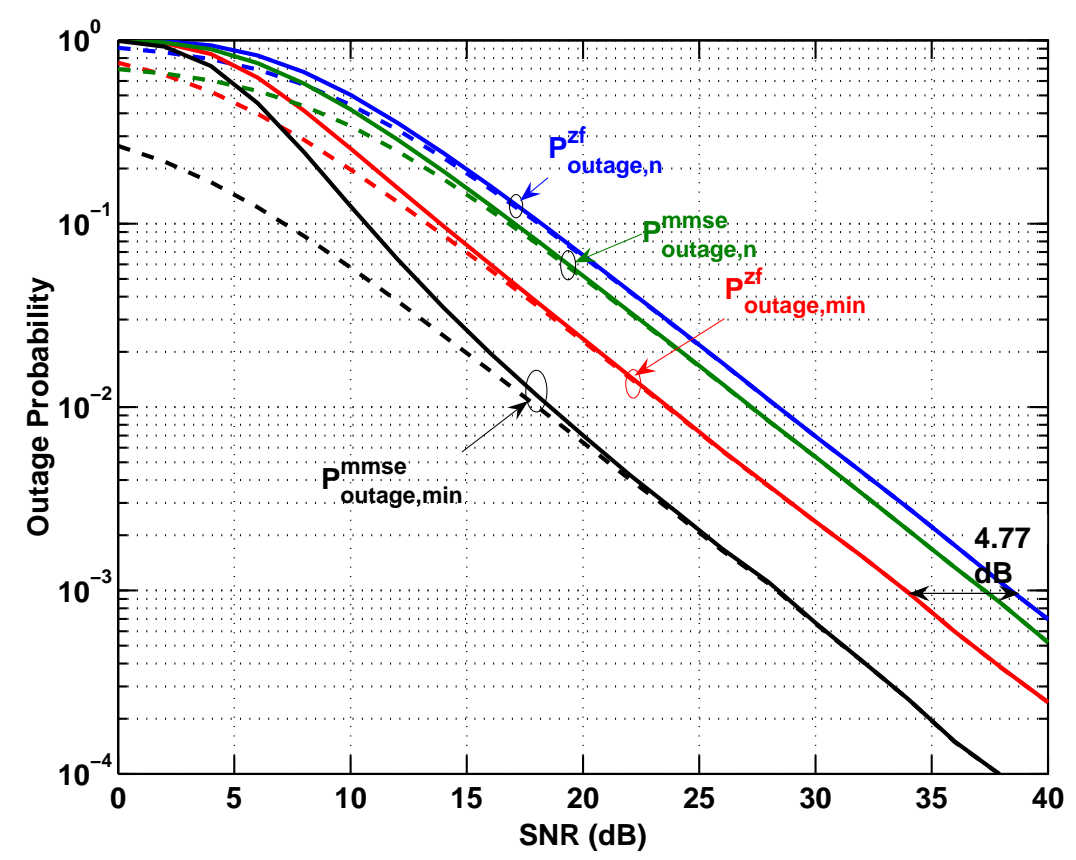

Fig. 5. Outage probabilities $P_{o u t, 1}^{z f}, P_{o u t, 1}^{m m s e}, P_{\mathrm{out}, \mathrm{min}}^{\mathrm{zf}}$, and $P_{\mathrm{out}, \mathrm{min}}^{\mathrm{mmse}}$. The solid lines are the true values and the dash lines are lower bounds. $M=N=3$. The result is obtained via averaging over $10^{6}$ Monte Carlo trials of the channel matrix.

Figure 6 adopts the same simulation layout as that of Figure 5 except that the channel dimensionality is changed to $M=N=5$. As we can see from Figure 6 , the gap between $P_{\text {out,min }}^{\mathrm{zf}}$ and $P_{\text {out }, 1}^{z f}$ is $10 \log _{10} N=6.99 \mathrm{~dB}$, and the SNR gain of $P_{\text {out,min }}^{\text {mmse }}$ over $P_{\text {out }, 1}^{m m s e}$ is even more significant compared to Figure 5. This result agrees with our analysis of (133) and (134).

In the final example, we consider an i.i.d. Rayleigh channel with $M=N=4$. We compare the outage probabilities of the strongest substreams $\left(P_{\text {out,min }}^{\mathrm{zf}}, P_{\text {out,min }}^{\mathrm{mmse}}\right)$, their lower bounds $\left(P_{\text {out,min }}^{\mathrm{zf}}, \underline{P_{\text {out,min }}^{\mathrm{mmse}}}\right)$, along with the outage probabilities of ZF-VB and MMSE-VB with optimal detection ordering. It is seen from Figure 7 that the lower bounds to $P_{\text {out,min }}^{\mathrm{zf}}$ and $P_{\text {out,min }}^{\text {mmse }}$ are also tight lower bounds to the outage probability of ZF-VB and MMSE-VB with optimal detection ordering, respectively. Hence theorem VI.2 can be used to predict the SNR gain of ordered detection in V-BLAST architecture. This example also validates Corollary V.4; the detection ordering cannot improve the diversity gain, which is $M-N+1=1$ in this example, although MMSE-VB manifests higher diversity gain in the low to moderate SNR regime $(\mathrm{snr} \leq 15 \mathrm{~dB})$. 


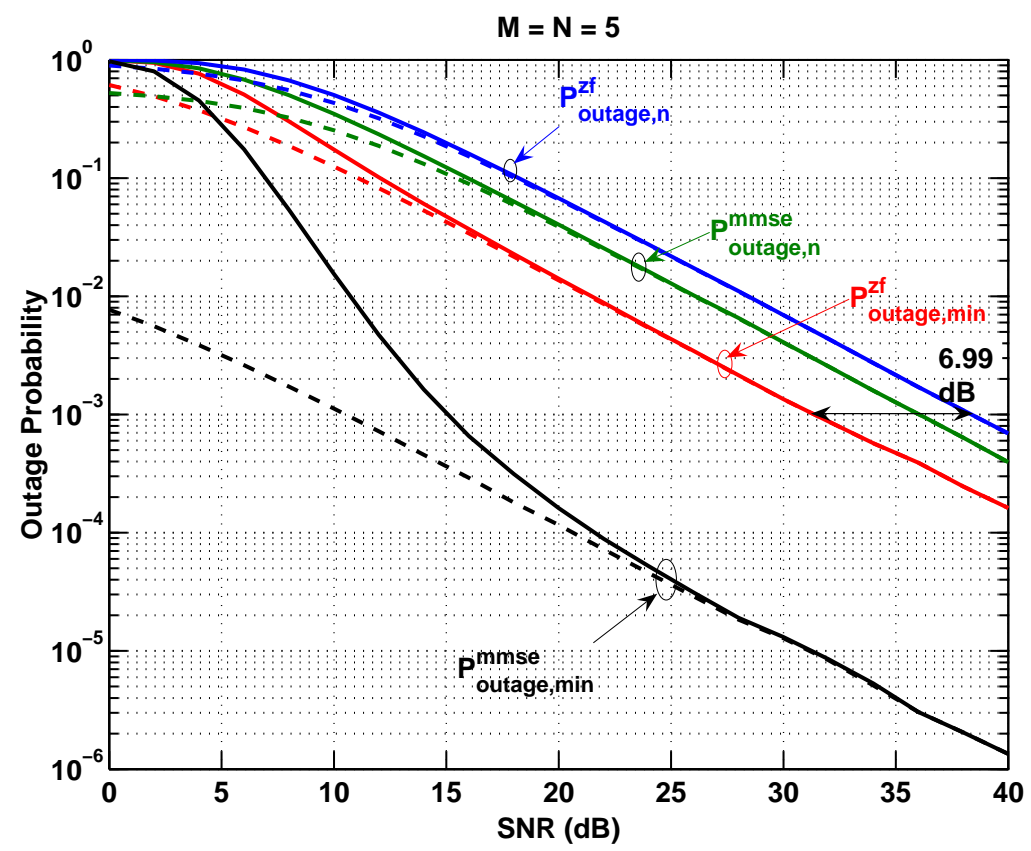

Fig. 6. Outage probabilities $P_{\text {out }, 1}^{z f}, P_{\text {out }, 1}^{m m s e}, P_{\text {out,min }}^{\mathrm{zf}}$, and $P_{\text {out }, \text { min }}^{\mathrm{mmse}}$. The solid lines are the true values and the dash lines are lower bounds. $M=N=5$.

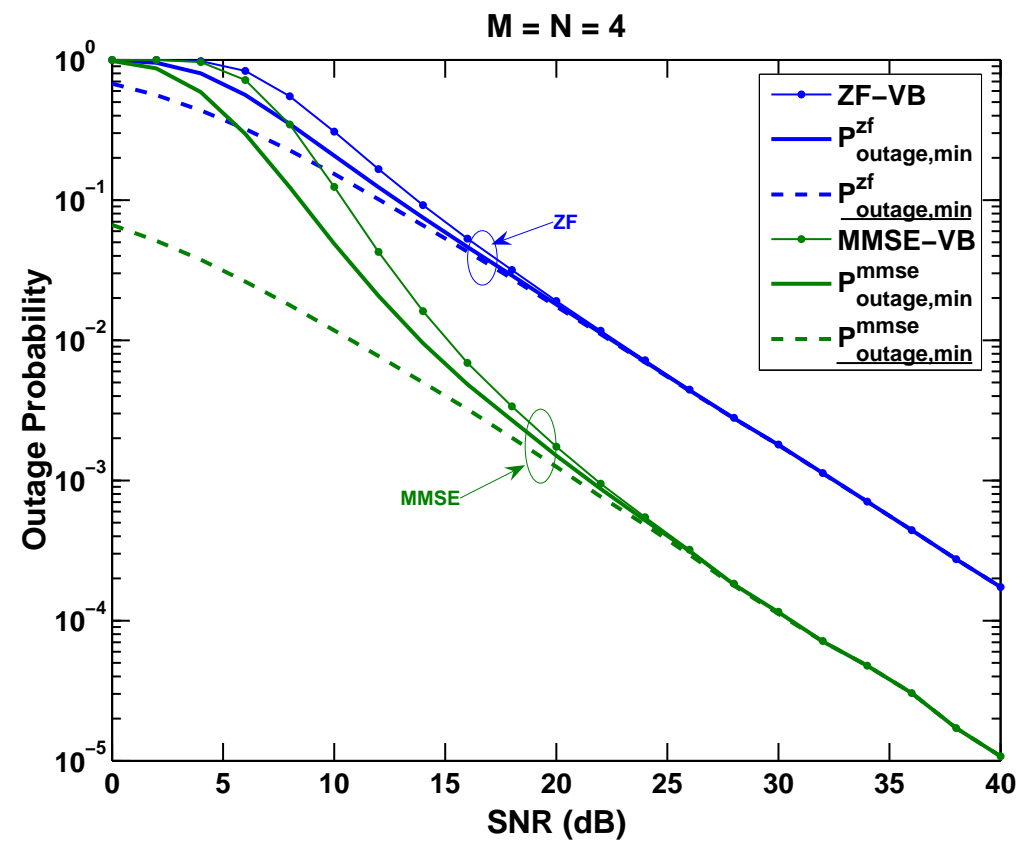

Fig. 7. Outage probabilities $P_{\text {out,min }}^{\mathrm{zf}}$, and $P_{\text {out,min }}^{\text {mmse }}$ ZF-VB and MMSE-VB with optimal ordering. The dash lines are the lower bounds $\underline{P_{\mathrm{out}, \mathrm{min}}^{\mathrm{zf}}}$ and $\underline{P_{\mathrm{out}, \mathrm{min}}^{\mathrm{mmse}}} \cdot M=N=4$. The result is obtained via averaging over $10^{7}$ Monte Carlo trials.

\section{Conclusions}

In this paper, we have analyzed the performances of the zero forcing (ZF) and minimal mean squared error (MMSE) equalizers applied to an $M \times N$ wireless multi-input multi-output (MIMO) systems, 
in terms of output SNR, uncoded error and outage probabilities, diversity-multiplexing (D-M) gain tradeoff, and SNR gain. We show that there is a gap between the output SNRs of ZF and MMSE equalizers, which converges with probability one to a random variable of scaled $\mathcal{F}$-distribution as input SNR goes to infinity. Based on this result, we can accurately approximate the uncoded error probability of MMSE equalizer via numerical integration rather than time-consuming Monte-Carlo simulations. For coded systems, we show that although given fixed target rate MMSE has a nonvanishing SNR gain over ZF, the $\epsilon$-outage capacities of MMSE and ZF coincide in the asymptotically high SNR regime. We also prove that even with perfect coding across the $N$ substreams, the D-M gain tradeoff of the MIMO system using either ZF or MMSE equalizer is $d(r)=(M-N+1)\left(1-\frac{r}{N}\right)$. As an important corollary, we prove that the V-BLAST equalizer (vertical Bell Labs layered Space-Time) has a maximal diversity gain of $M-N+1$ even with optimal order detection. Moreover, we show that for the ZF equalizer, the strongest substream has $10 \log _{10} N \mathrm{~dB}$ SNR gain over an average one. For MMSE, this SNR gain is much larger than even that. This analysis also quantifies the SNR gain of applying ordered detection in V-BLAST architecture.

\section{Appendix A}

\section{ProOF OF LEMMA III.2}

According to the matrix inverse lemma,

$$
\left(\mathbf{H}^{*} \mathbf{H}+\frac{1}{\mathrm{snr}} \mathbf{I}\right)^{-1}=\operatorname{snr}\left[\mathbf{I}-\mathbf{H}^{*}\left(\frac{1}{\mathrm{snr}} \mathbf{I}+\mathbf{H H}^{*}\right)^{-1} \mathbf{H}\right] .
$$

Therefore we can rewrite the MMSE filter matrix given in (11) by

$$
\begin{aligned}
\mathbf{W}_{\mathrm{mmse}} & =\operatorname{snr}\left[\mathbf{I}-\mathbf{H}^{*}\left(\frac{1}{\mathrm{snr}} \mathbf{I}+\mathbf{H H}^{*}\right)^{-1} \mathbf{H}\right] \mathbf{H}^{*} \\
& =\mathbf{H}^{*}\left(\mathbf{H} \mathbf{H}^{*}+\frac{1}{\mathrm{snr}} \mathbf{I}\right)^{-1} .
\end{aligned}
$$

Denote $\mathbf{w}_{n}^{*}$ the $n$th row of $\mathbf{W}_{\text {mmse }}$, which is the MMSE nulling vector for the $n$th substream. Then

$$
\mathbf{w}_{n}=\left(\mathbf{H H}^{*}+\frac{1}{\mathrm{snr}} \mathbf{I}\right)^{-1} \mathbf{h}_{n} \propto\left(\mathbf{H}_{n} \mathbf{H}_{n}^{*}+\frac{1}{\mathrm{snr}} \mathbf{I}\right)^{-1} \mathbf{h}_{n},
$$

where $\mathbf{h}_{n}$ and $\mathbf{H}_{n}$ are defined as in (14). If we normalize $\mathbf{w}_{n}$ such that $\mathbf{w}_{n}^{*} \mathbf{h}_{n}=1$, then

$$
\mathbf{w}_{n}=\frac{\left(\mathbf{H}_{n} \mathbf{H}_{n}^{*}+\frac{1}{\mathrm{snr}} \mathbf{I}\right)^{-1} \mathbf{h}_{n}}{\mathbf{h}_{n}^{*}\left(\mathbf{H}_{n} \mathbf{H}_{n}^{*}+\frac{1}{\mathrm{snr}} \mathbf{I}\right)^{-1} \mathbf{h}_{n}} .
$$


Without loss of generality, we assume here that $\sigma_{x}^{2}=1$ and $\sigma_{z}^{2}=\frac{1}{\operatorname{snr}}$. Applying $\mathbf{w}_{n}$ to the received data vector $\mathbf{y}$ (cf. (1)), the power of noise is

$$
P_{\mathrm{ns}, \mathrm{mmse}}=\frac{1}{\mathrm{snr}}\left\|\mathbf{w}_{n}\right\|^{2}=\frac{\mathbf{h}_{n}^{*}\left(\mathbf{H}_{n} \mathbf{H}_{n}^{*}+\frac{1}{\mathrm{snr}} \mathbf{I}\right)^{-2} \mathbf{h}_{n}}{\mathrm{snr}\left|\mathbf{h}_{n}^{*}\left(\mathbf{H}_{n} \mathbf{H}_{n}^{*}+\frac{1}{\mathrm{snr}} \mathbf{I}\right)^{-1} \mathbf{h}_{n}\right|^{2}},
$$

and the sum power of noise and interference from the other $N-1$ substreams is

$$
P_{\mathrm{ns}, \mathrm{mmse}}+P_{\mathrm{intf}, \mathrm{mmse}}=\mathbf{w}_{n}^{*}\left(\mathbf{H}_{n} \mathbf{H}_{n}^{*}+\frac{1}{\mathrm{snr}} \mathbf{I}\right) \mathbf{w}=\frac{1}{\mathbf{h}_{n}^{*}\left(\mathbf{H}_{n} \mathbf{H}_{n}^{*}+\frac{1}{\mathrm{snr}} \mathbf{I}\right)^{-1} \mathbf{h}_{n}}
$$

¿From (140) and (139), we have the ratio

$$
\frac{P_{\mathrm{ns}, \mathrm{mmse}}+P_{\mathrm{intf}, \mathrm{mmse}}}{P_{\mathrm{ns}, \mathrm{mmse}}}=\frac{\operatorname{snr}_{n}^{*}\left(\mathbf{H}_{n} \mathbf{H}_{n}^{*}+\frac{1}{\mathrm{snr}} \mathbf{I}\right)^{-1} \mathbf{h}_{n}}{\mathbf{h}_{n}^{*}\left(\mathbf{H}_{n} \mathbf{H}_{n}^{*}+\frac{1}{\mathrm{snr}} \mathbf{I}\right)^{-2} \mathbf{h}_{n}} .
$$

Denote $\mathbf{H}_{n}=\mathbf{U}_{n} \boldsymbol{\Lambda}_{n} \mathbf{V}_{n}^{*}$ the SVD of $\mathbf{H}_{n}$, where $\mathbf{U}_{n} \in \mathbb{C}^{M \times(N-1)}$ and $\boldsymbol{\Lambda}_{n} \in \mathbb{R}^{(N-1) \times(N-1)}$. Then the $\mathrm{SVD}$ of $\mathbf{H}_{n} \mathbf{H}_{n}^{*}+\frac{1}{\mathrm{snr}} \mathbf{I}$ is

$$
\mathbf{H}_{n} \mathbf{H}_{n}^{*}+\frac{1}{\mathrm{snr}} \mathbf{I}=\left[\mathbf{U}_{n} \vdots \overline{\mathbf{U}}_{n}\right]\left[\begin{array}{cc}
\boldsymbol{\Lambda}_{n}^{2}+\frac{1}{\mathrm{snr}} \mathbf{I}_{N-1} & \mathbf{0} \\
\mathbf{0} & \frac{1}{\mathrm{snr}} \mathbf{I}_{M-N+1}
\end{array}\right]\left[\mathbf{U}_{n} \vdots \overline{\mathbf{U}}_{n}\right]^{*} .
$$

Now we can rewrite

$$
\mathbf{h}_{n}^{*}\left(\mathbf{H}_{n} \mathbf{H}_{n}^{*}+\frac{1}{\mathrm{snr}} \mathbf{I}\right)^{-1} \mathbf{h}_{n}=\mathbf{h}_{n}^{*} \mathbf{U}_{n}\left(\boldsymbol{\Lambda}_{n}^{2}+\frac{1}{\mathrm{snr}} \mathbf{I}\right)^{-1} \mathbf{U}_{n}^{*} \mathbf{h}_{n}+\operatorname{snrh}_{n}^{*} \overline{\mathbf{U}}_{n} \overline{\mathbf{U}}_{n}^{*} \mathbf{h}_{n},
$$

and

$$
\mathbf{h}_{n}^{*}\left(\mathbf{H}_{n} \mathbf{H}_{n}^{*}+\frac{1}{\mathrm{snr}} \mathbf{I}\right)^{-2} \mathbf{h}_{n}=\mathbf{h}_{n}^{*} \mathbf{U}_{n}\left(\boldsymbol{\Lambda}_{n}^{2}+\frac{1}{\mathrm{snr}} \mathbf{I}\right)^{-2} \mathbf{U}_{n}^{*} \mathbf{h}_{n}+\operatorname{snr}^{2} \mathbf{h}_{n}^{*} \overline{\mathbf{U}}_{n} \overline{\mathbf{U}}_{n}^{*} \mathbf{h}_{n} .
$$

Applying (143) and (144) the fact that $\overline{\mathbf{U}}_{n} \overline{\mathbf{U}}_{n}^{*}=\mathbf{P}_{\mathbf{H}_{n}}^{\perp}$, we obtain from (141) that

$$
\frac{P_{\mathrm{ns}, \mathrm{mmse}}+P_{\text {intf,mmse }}}{P_{\mathrm{ns}, \mathrm{mmse}}}=\frac{\mathbf{h}_{n}^{*} \mathbf{P}_{\mathbf{H}_{n}}^{\perp} \mathbf{h}_{n}+\mathrm{snr}^{-1} \mathbf{h}_{n}^{*} \mathbf{U}_{n}\left(\boldsymbol{\Lambda}_{n}^{2}+\frac{1}{\mathrm{snr}} \mathbf{I}\right)^{-1} \mathbf{U}_{n}^{*} \mathbf{h}_{n}}{\mathbf{h}_{n}^{*} \mathbf{P}_{\mathbf{H}_{n}}^{\perp} \mathbf{h}_{n}+\mathrm{snr}^{-2} \mathbf{h}_{n}^{*} \mathbf{U}_{n}\left(\boldsymbol{\Lambda}_{n}^{2}+\frac{1}{\mathrm{snr}} \mathbf{I}\right)^{-2} \mathbf{U}_{n}^{*} \mathbf{h}_{n}}
$$

and

$$
\begin{aligned}
\frac{P_{\text {intf,mmse }}}{P_{\mathrm{ns}, \mathrm{mmse}}} & =\frac{\mathrm{snr}^{-1} \mathbf{h}_{n}^{*} \mathbf{U}_{n}\left(\boldsymbol{\Lambda}_{n}^{2}+\frac{1}{\mathrm{snr}} \mathbf{I}\right)^{-1} \mathbf{U}_{n}^{*} \mathbf{h}_{n}-\mathrm{snr}^{-2} \mathbf{h}_{n}^{*} \mathbf{U}_{n}\left(\boldsymbol{\Lambda}_{n}^{2}+\frac{1}{\mathrm{snr}} \mathbf{I}\right)^{-2} \mathbf{U}_{n}^{*} \mathbf{h}_{n}}{\mathbf{h}_{n}^{*} \mathbf{P}_{\mathbf{H}_{n}}^{\perp} \mathbf{h}_{n}+\mathrm{snr}^{-2} \mathbf{h}_{n}^{*} \mathbf{U}_{n}\left(\boldsymbol{\Lambda}_{n}^{2}+\frac{1}{\mathrm{snr}} \mathbf{I}\right)^{-2} \mathbf{U}_{n}^{*} \mathbf{h}_{n}} \\
& \lesssim \frac{\mathbf{h}_{n}^{*} \mathbf{U}_{n}\left(\boldsymbol{\Lambda}_{n}^{2}+\frac{1}{\mathrm{snr}} \mathbf{I}\right)^{-1} \mathbf{U}_{n}^{*} \mathbf{h}_{n}}{\mathrm{snr} \cdot \mathbf{h}_{n}^{*} \mathbf{P}_{\mathbf{H}_{n}}^{\perp} \mathbf{h}_{n}}
\end{aligned}
$$

It is easy to see that the upper bound of (147) is asymptotically tight as snr $\rightarrow \infty$. We note that the numerator and the denominator in (147) are exactly $\eta_{\mathrm{snr}, n}$ (see (26)) and $\rho_{\mathrm{zf}, n}$ (see (15)), respectively. The Lemma is proven. 


\section{Appendix B}

Proof of Lemma VI.1

We first prove (121). According to (117), we can rewrite

$$
P_{\text {out }, n}^{\mathrm{zf}}(R, \mathrm{snr})=\mathbb{P}\left(\frac{\lambda_{N}^{2}}{\left|v_{n N}\right|^{2}} \cdot \frac{1}{1+\frac{\lambda_{N}^{2}}{\left|v_{n N}\right|^{2}} \sum_{i=1}^{N-1} \frac{\left|v_{n i}\right|^{2}}{\lambda_{i}^{2}}}<\frac{2^{R}-1}{\mathrm{snr}}\right) .
$$

Because $\lambda_{n}^{2}$ 's are in non-increasing order, we have

$$
\sum_{i=1}^{N-1} \frac{\left|v_{n i}\right|^{2}}{\lambda_{n}^{2}} \leq \sum_{i=1}^{N-1} \frac{\left|v_{n i}\right|^{2}}{\lambda_{N-1}^{2}} \leq \frac{1}{\lambda_{N-1}^{2}} .
$$

Combining (148) and (149) yields

$$
\begin{aligned}
P_{\mathrm{out}, n}^{\mathrm{zf}}(R, \mathrm{snr}) & \leq \mathbb{P}\left(\frac{\lambda_{N}^{2}}{\left|v_{n N}\right|^{2}} \cdot \frac{1}{1+\frac{\lambda_{N}^{2}}{\left|v_{n N}\right|^{2}} \frac{1}{\lambda_{N-1}^{2}}}<\frac{2^{R}-1}{\mathrm{snr}}\right) \\
& =\underline{P_{\text {out }, n}^{\mathrm{zf}}}(R, \mathrm{snr})+\mathbb{P}\left(\frac{\lambda_{N}^{2}}{\left|v_{n N}\right|^{2}}>\frac{2^{R}-1}{\mathrm{snr}}, \lambda_{N-1}^{2}<\frac{\frac{\lambda_{N}^{2}}{\left|v_{n N}\right|^{2}}}{\frac{\lambda_{N}^{2}}{\left|v_{n N}^{2}\right|} \frac{\mathrm{snr}}{2^{R}-1}-1}\right) .
\end{aligned}
$$

We now focus on the second term of the right hand side of (151)

$$
\begin{aligned}
& \mathbb{P}\left(\frac{\lambda_{N}^{2}}{\left|v_{n N}\right|^{2}}>\frac{2^{R}-1}{\mathrm{snr}}, \lambda_{N-1}^{2}<\frac{\frac{\lambda_{N}^{2}}{\left|v_{n N}\right|^{2}}}{\frac{\lambda_{N}^{2}}{\left|v_{n N}^{2}\right|} \frac{\mathrm{snr}}{2^{R}-1}-1}\right) \\
= & \mathbb{P}\left(\frac{2^{R}-1}{\mathrm{snr}}<\frac{\lambda_{N}^{2}}{\left|v_{n N}\right|^{2}}<\left(1+\frac{1}{\log \mathrm{snr}}\right) \frac{2^{R}-1}{\mathrm{snr}}, \lambda_{N-1}^{2}<\frac{\frac{\lambda_{N}^{2}}{\left|v_{n N}\right|^{2}}}{\frac{\lambda_{N}^{2}}{\left|v_{n N}^{2}\right|} \frac{\mathrm{snr}}{2^{R}-1}-1}\right) \\
& +\mathbb{P}\left(\frac{\lambda_{N}^{2}}{\left|v_{n N}\right|^{2}}>\left(1+\frac{1}{\log \mathrm{snr}}\right) \frac{2^{R}-1}{\mathrm{snr}}, \lambda_{N-1}^{2}<\frac{\frac{\lambda_{N}^{2}}{\left|v_{n N}\right|^{2}}}{\frac{\lambda_{N}^{2}}{\left|v_{n N}^{2}\right|} \frac{\mathrm{snr}}{2^{R}-1}-1}\right) \\
\leq & \mathbb{P}\left(\frac{2^{R}-1}{\mathrm{snr}}<\frac{\lambda_{N}^{2}}{\left|v_{n N}\right|^{2}}<\left(1+\frac{1}{\log \mathrm{snr}}\right) \frac{2^{R}-1}{\mathrm{snr}}\right)+\mathbb{P}\left(\lambda_{N-1}^{2}<(1+\log \mathrm{snr}) \frac{2^{R}-1}{\mathrm{snr}}\right)
\end{aligned}
$$

To obtain (154) from (153), we have used the following two facts. First,

$$
\begin{aligned}
& \mathbb{P}\left(\frac{2^{R}-1}{\mathrm{snr}}<\frac{\lambda_{N}^{2}}{\left|v_{n N}\right|^{2}}<\left(1+\frac{1}{\log \mathrm{snr}}\right) \frac{2^{R}-1}{\mathrm{snr}}, \lambda_{N-1}^{2}<\frac{\frac{\lambda_{N}^{2}}{\left|v_{n N}\right|^{2}}}{\frac{\lambda_{N}^{2}}{\left|v_{n N}^{2}\right|} \frac{\mathrm{snr}}{2^{R}-1}-1}\right) \\
\leq & \mathbb{P}\left(\frac{2^{R}-1}{\mathrm{snr}}<\frac{\lambda_{N}^{2}}{\left|v_{n N}\right|^{2}}<\left(1+\frac{1}{\log \mathrm{snr}}\right) \frac{2^{R}-1}{\mathrm{snr}}\right) .
\end{aligned}
$$

Second, because

$$
\frac{\frac{\lambda_{N}^{2}}{\left|v_{n N}\right|^{2}}}{\frac{\lambda_{N}^{2}}{\left|v_{n N}^{2}\right|} \frac{\mathrm{snr}}{2^{R}-1}-1}
$$


is a decreasing function of $\frac{\lambda_{N}^{2}}{\left|v_{n N}\right|^{2}}$, for

$$
\frac{\lambda_{N}^{2}}{\left|v_{n N}\right|^{2}}>\left(1+\frac{1}{\log \mathrm{snr}}\right) \frac{2^{R}-1}{\mathrm{snr}}
$$

we have

$$
\frac{\frac{\lambda_{N}^{2}}{\left|v_{n N}\right|^{2}}}{\frac{\lambda_{N}^{2}}{\left|v_{n N}^{2}\right|} \frac{\mathrm{snr}}{2^{R}-1}-1}<\frac{\left(1+\frac{1}{\log \mathrm{snr}}\right) \frac{2^{R}-1}{\mathrm{snr}}}{\left(1+\frac{1}{\log \mathrm{snr}}\right) \frac{2^{R}-1}{\mathrm{snr}} \frac{\mathrm{snr}}{2^{R}-1}-1}=(1+\log \mathrm{snr}) \frac{2^{R}-1}{\mathrm{snr}} .
$$

Hence,

$$
\begin{aligned}
& \mathbb{P}\left(\frac{\lambda_{N}^{2}}{\left|v_{n N}\right|^{2}}>\left(1+\frac{1}{\log \mathrm{snr}}\right) \frac{2^{R}-1}{\mathrm{snr}}, \lambda_{N-1}^{2}<\frac{\frac{\lambda_{N}^{2}}{\left|v_{n N}\right|^{2}}}{\frac{\lambda_{N}^{2}}{\left|v_{n N}^{2}\right|} \frac{\mathrm{snr}}{2^{R}-1}-1}\right) \\
\leq & \mathbb{P}\left(\frac{\lambda_{N}^{2}}{\left|v_{n N}\right|^{2}}>\left(1+\frac{1}{\log \mathrm{snr}}\right) \frac{2^{R}-1}{\mathrm{snr}}, \lambda_{N-1}^{2}<(1+\log \mathrm{snr}) \frac{2^{R}-1}{\mathrm{snr}}\right) \\
\leq & \mathbb{P}\left(\lambda_{N-1}^{2}<(1+\log \mathrm{snr}) \frac{2^{R}-1}{\mathrm{snr}}\right) .
\end{aligned}
$$

Therefore using the inequalities (155) and (156), we obtain (154) from (153).

Combining (151) and (154), we have

$$
P_{\text {out }, n}^{\mathrm{zf}}(R, \mathrm{snr}) \leq \mathbb{P}\left(\frac{\lambda_{N}^{2}}{\left|v_{n N}\right|^{2}}<\left(1+\frac{1}{\log \mathrm{snr}}\right) \frac{2^{R}-1}{\mathrm{snr}}\right)+\mathbb{P}\left(\lambda_{N-1}^{2}<(1+\log \mathrm{snr}) \frac{2^{R}-1}{\mathrm{snr}}\right)
$$

According to Theorem II.3, as snr $\rightarrow \infty$, the second term of the above equation

$$
\mathbb{P}\left(\lambda_{N-1}^{2}<(1+\log \operatorname{snr}) \frac{2^{R}-1}{\mathrm{snr}}\right)=\left(\frac{\left(2^{R}-1\right)(1+\log \mathrm{snr})}{\mathrm{snr}}\right)^{2(M-N+2)+o(1)},
$$

while the first term

$$
\begin{aligned}
\mathbb{P}\left(\frac{\lambda_{N}^{2}}{\left|v_{n N}\right|^{2}}<\left(1+\frac{1}{\log \mathrm{snr}}\right) \frac{2^{R}-1}{\mathrm{snr}}\right) & >\mathbb{P}\left(\lambda_{N}^{2}<c\left(1+\frac{1}{\log \mathrm{snr}}\right) \frac{2^{R}-1}{\mathrm{snr}}\right) \mathbb{P}\left(\left|v_{n N}\right|^{2}>c\right) \\
& =K \cdot\left(\frac{2^{R}-1}{\mathrm{snr}}\left(1+\frac{1}{\log \mathrm{snr}}\right)\right)^{M-N+1+o(1)},
\end{aligned}
$$

where $c$ is some finite positive constant, say $c=\frac{1}{2 N}$, and $K=\mathbb{P}\left(\left|v_{n N}\right|^{2}>c\right) \cdot c^{M-N+1}$ is also a finite positive constant.

It follows from (158) and (159) that given fixed $R$,

$$
\lim _{\mathrm{snr} \rightarrow \infty} \frac{\mathbb{P}\left(\lambda_{N-1}^{2}<(1+\log \mathrm{snr}) \frac{2^{R}-1}{\mathrm{snr}}\right)}{\mathbb{P}\left(\frac{\lambda_{N}^{2}}{\left|v_{n N}\right|^{2}}<\frac{2^{R}-1}{\mathrm{snr}}\right)}=0 .
$$


Hence

$$
\begin{aligned}
\lim _{\mathrm{snr} \rightarrow \infty} \frac{P_{\text {out }, n}^{\mathrm{zf}}(R, \mathrm{snr})}{\frac{P_{\text {out }, n}^{\mathrm{zf}}(R, \mathrm{snr})}{\mathrm{s}}} & \leq \lim _{\mathrm{snr} \rightarrow \infty} \frac{\mathbb{P}\left(\frac{\lambda_{N}^{2}}{\left|v_{n N}\right|^{2}}<\left(1+\frac{1}{\log \mathrm{snr}}\right) \frac{2^{R}-1}{\mathrm{snr}}\right)+\mathbb{P}\left(\lambda_{N-1}^{2}<(1+\log \mathrm{snr}) \frac{2^{R}-1}{\mathrm{snr}}\right)}{\mathbb{P}\left(\frac{\lambda_{N}^{2}}{\left|v_{n N}\right|^{2}}<\frac{2^{R}-1}{\mathrm{snr}}\right)} \\
& =\lim _{\mathrm{snr} \rightarrow \infty} \frac{\mathbb{P}\left(\frac{\lambda_{N}^{2}}{\left|v_{n N}\right|^{2}}<\left(1+\frac{1}{\log \mathrm{snr}}\right) \frac{2^{R}-1}{\mathrm{snr}}\right)}{\mathbb{P}\left(\frac{\lambda_{N}^{2}}{\left|v_{n N}\right|^{2}}<\frac{2^{R}-1}{\mathrm{snr}}\right)} \\
& =\lim _{\mathrm{snr} \rightarrow \infty}\left(1+\frac{1}{\log \mathrm{snr}}\right)^{M-N+1}=1
\end{aligned}
$$

On the other hand, according to (118)

$$
\lim _{\mathrm{snr} \rightarrow \infty} \frac{P_{\text {out }, n}^{\mathrm{zf}}(R, \mathrm{snr})}{\underline{P_{\text {out }, n}^{\mathrm{zf}}}(R, \mathrm{snr})} \geq 1 .
$$

Combining (160) and (161), we have proven (121).

The techniques used above in the proof for (121) can be equally applied to prove (122). We omit it for simplicity.

\section{ACKnowledgement}

The first author thanks Dr. X. Zheng for the helpful discussions at the early stage of this research work.

\section{REFERENCES}

[1] J. G. Proakis, Digital Communications. McGraw-Hill Inc., Third Edition, 1995.

[2] G. J. Foschini, G. D. Golden, R. A. Valenzuela, and P. W. Wolniansky, "Simplified processing for high spectral efficiency wireless communication employing multiple-element arrays," Wireless Personal Communications, vol. 6, pp. 311-335, March 1999.

[3] G. Ginis and J. M. Cioffi, "On the relationship between V-BLAST and the GDFE," IEEE Communications Letters, vol. 5, pp. 364-366, September 2001.

[4] D. Palomar, J. Cioffi, and M. Lagunas, "Joint Tx-Rx beamforming design for multicarrier MIMO channels: A unified framework for convex optimization," IEEE Transactions on Signal Processing, vol. 51, pp. 2381-2401, September 2003.

[5] Y. Jiang, J. Li, and W. Hager, "Uniform channel decomposition for MIMO communications," IEEE Transactions on Signal Processing, vol. 53, pp. 4283 - 4294, November 2005.

[6] L. Zheng and D. Tse, "Diversity and multiplexing: A fundamental tradeoff in multiple-antenna channels," IEEE Transactions on Information Theory, vol. 49, pp. 1073-1096, May 2003.

[7] G. Casella and R. L. Berger, Statistical Inference. Duxbury Press, 2001.

[8] J. Zhang, E. K. P. Chong, and D. N. C. Tse, "Output mai distribution of linear mmse multiuser receivers in ds-cdma systems," IEEE Transactions on Information Theory, vol. 47, p. 2001, March 2001. 
[9] D. Guo, S. Verdu, and L. K. Rasmussen, "Asymptotic normality of linear multiuser receiver outputs," IEEE Trans. Inform. Theory, vol. 48, pp. 3080-3095, December 2002.

[10] H. V. Poor and S. Verdu, "Probability of error in MMSE multiuser detection," IEEE Transactions on Information Theory, vol. 43, pp. 858-871, May 1997.

[11] Y. Jiang, X. Zheng, and J. Li, "Asymptotic analysis of V-BLAST," Proceedings of IEEE GlobeCom, St. Louis, MO, November 2005.

[12] P. Li, D. Paul, R. Narasimhan, and J. Cioffi, "On the distribution of SINR for the MMSE MIMO receiver and performance analysis," IEEE Transactions on Information Theory, vol. 52, pp. 271-286, January 2006.

[13] N. Prasad and M. K. Varanasi, "Analysis of decision feedback detection for MIMO rayleigh fading channels and the optimization of rate and power allocations," IEEE Transactions on Information Theory, vol. 50, pp. 1009-1025, June 2004.

[14] S. Loyka and F. Gagon, "Performance analysis of the V-BLAST algorithm: an analytical approach," IEEE Transactions on Wireless Communications, vol. 3, pp. 1326-1337, July 2004.

[15] S. Loyka and F. Gagon, "Analytical framework for outage and BER analysis of the V-BLAST algorithm," Int. Zurich Seminar on Communications (IZS), pp. 120-123, Feb. 18-20 2004.

[16] M. O. Damen, K. Abed-Meriam, and S. Burykh, "Iterative QR detection for an uncoded space-time communication architecture," Proc. Allerton Conf. Communications, Control, and Computing, October 2000.

[17] X. Zhang and S. Kung, "Capacity analysis for parallel and sequential MIMO equalizers," IEEE Transactions on Signal Processing, vol. 51, pp. 2989-3002, November 2003.

[18] B. Hochwald and S. Vishwanath, "Space-time multiple access: linear growth in the sum rate," Proceedings: Allerton Conference on Communications, Control, and Computing, Monticello, IL, October 2002.

[19] N. Jindal, "High SNR analysis of MIMO broadcast channels," Proc. IEEE Int. Symp. Information Theory, Adelaide, Australia, Sept. 2005.

[20] D. Tse and P. Viswanath, Fundamentals of Wireless Communications. Cambridge Press, 2005.

[21] R. A. Horn and C. R. Johnson, Matrix Analysis. Cambridge: Cambridge University Press, 1985.

[22] T. W. Anderson, An Introduction to Multivariate Statistical Analysis. second edition, John Wiley and Sons, Inc., 1984.

[23] A. M. Tulino and S. Verdu, Random Matrix Theory and Wireless Communications. Hanover, MA 02339, USA: now Publishers Inc., 2004.

[24] G. H. Golub and C. F. Van Loan, Matrix Computations. Baltimore, MD: Johns Hopkins University Press, 1983.

[25] H. L. Van Trees, Detection, Estimation, and Modulation Theory, Part I. New York, NY: John Wiley and Sons, Inc., 1968.

[26] J. Craig, "New, simple and exact result for calculating the probability of error for two-dimensional signal constellations," Proc. Milcom, 1991.

[27] K. Cho and D. Yoon, "On the general BER expression of one and two-dimensional amplitude modulations," IEEE Transactions on Communications, vol. 50, pp. 1074-1080, July 2002.

[28] S. M. Ross, Introduction to Probability Models, Seventh Edition. HarCour Academic Press, 2000.

[29] M. Varanasi and T. Guess, "Optimum decision feedback multiuser equalization with successive decoding achieves 
the total capacity of the Gaussian multiple-access channel," Proceedings of the Thirty-First Asilomar Conference on Signals, Systems and Computers, vol. 2, pp. 1405 - 1409, Nov 2-5 1997.

[30] B. Hassibi, "A fast square-root implementation for BLAST," Thirty-Fourth Asilomar Conf. Signals, Systems and Computers, pp. 1255-1259, November 2000.

[31] N. Prasad and M. K. Varanasi, "Outage capacities of space-time architecture," Information Theory Workshop, San Antonio, Texas, October 24-29 2004.

[32] M. Varanasi, "Group detection for synchronous gaussian code-division multiple-access channels," IEEE Transactions on Information Theory, vol. 41, pp. 1083-1096, July 1995.

[33] H. Zhang, H. Dai, Q. Zhou, and B. L. Hughes, "On the diversity-multiplexing tradeoff for ordered SIC receivers over MIMO channels," IEEE International Conference on Communications (ICC), Istanbul, Turkey, June 2006. 